\title{
Predictive pore-scale modeling of two-phase flow in mixed wet media
}

\author{
Per H. Valvatne ${ }^{1}$ and Martin J. Blunt \\ Department of Earth Science and Engineering, Imperial College London, London, UK \\ Received 26 August 2003; revised 3 March 2004; accepted 16 April 2004; published 15 July 2004.
}

[1] We show how to predict flow properties for a variety of porous media using pore-scale modeling with geologically realistic networks. Starting with a network representation of Berea sandstone, the pore size distribution is adjusted to match capillary pressure for different media, keeping the rank order of pore sizes and the network topology fixed. Then predictions of single and multiphase properties are made with no further adjustment of the model. We successfully predict relative permeability and oil recovery for water wet, oil wet, and mixed wet data sets. For water flooding we introduce a method for assigning contact angles to match measured wettability indices. The aim of this work is not simply to match experiments but to use easily acquired data to predict difficult to measure properties. Furthermore, the variation of these properties in the field, due to wettability trends and different pore structures, can now be predicted reliably. INDEX TERMS: 5139 Physical Properties of Rocks: Transport properties; 5112 Physical Properties of Rocks: Microstructure; 5114 Physical Properties of Rocks: Permeability and porosity; 5199 Physical Properties of Rocks: General or miscellaneous; KEYWORDS: geologically realistic networks, multiphase flow, pore-scale modeling, relative permeability, wettability

Citation: Valvatne, P. H., and M. J. Blunt (2004), Predictive pore-scale modeling of two-phase flow in mixed wet media, Water Resour. Res., 40, W07406, doi:10.1029/2003WR002627.

\section{Introduction}

[2] Understanding multiphase flow at the pore-scale is of great importance in many fields such as hydrology, contaminant cleanup and petroleum engineering. In network modeling the void space of a rock is represented at the microscopic scale by a lattice of pores connected by throats. By applying rules that govern the transport and arrangement of fluids in pores and throats, macroscopic properties, for instance capillary pressure or relative permeability, can then be estimated across the network, which typically consists of several thousand pores and throats representing a rock sample of a few millimeters cubed.

[3] Until recently most networks were based on a regular lattice. The coordination number can vary depending on the chosen lattice (e.g., 5 for a honeycombed lattice or 6 for a regular cubic lattice). As has been noted by many authors [Chatzis and Dullien, 1977; Wilkinson and Willemsen, 1983] the coordination number will influence the network model behavior significantly, both in terms of breakthrough and relative permeability. In order to match the coordination number of a given rock sample, which typically is between 3 and 8 [Jerauld and Salter, 1990], it is possible to remove throats at random from a regular lattice [Dixit et al., 1997, 1999], hence reducing the connectivity. The pore and throat size distributions will also affect the estimated macroscopic properties. By adjusting the size distributions to match capillary pressure data, fairly good predictions of absolute

\footnotetext{
${ }^{1}$ Now at Shell International Exploration and Production B.V., Rijswijk, Netherlands.

Copyright 2004 by the American Geophysical Union. 0043-1397/04/2003WR002627\$09.00
}

and relative permeabilities have been reported for unsaturated soils [Fischer and Celia, 1999; Vogel, 2000].

[4] However, it seems clear that using a regular lattice structure to represent real porous media is not feasible if the network model is to have predictive capabilities. Even when mapping most of the morphological properties of a sandstone onto the lattice, Sok et al. [2002] were unable to predict satisfactorily the transport properties of a sandstone. Although Fischer and Celia [1999] managed to predict relative permeability for individual flooding cycles quite well, they were unable to predict hysteresis trends between different cycles.

[5] The use of networks derived from a real porous medium was pioneered by Bryant and Blunt [1992] and Bryant et al. [1993a, 1993b]. They extracted their networks from a random close packing of equally sized spheres where all sphere coordinates had been measured. Predictions of relative permeability, electrical conductivity and capillary pressure were compared successfully with experimental results from sand packs, bead packs and a simple sandstone.

[6] Øren and coworkers at Statoil have extended this approach to a wider range of sedimentary rocks [Bakke and Øren, 1997; Øren et al., 1998]. For more complex sandstones it is usually necessary to create first a 3-D voxel based representation of the pore space that should capture the statistics of the real rock. This can be generated directly using X-ray microtomography [Dunsmuir et al., 1991; Spanne et al., 1994], where the rock is imaged at resolutions of around a few microns, or by using some statistical [Adler and Thovert, 1998] or object-based [Bakke and Øren, 1997] reconstruction technique. From this voxel representation an equivalent network of pores and throats can be extracted [Lindquist et al., 1996; Bakke and Øren, 1997; Delerue and 
Perrier, 2002]. Individual elements are given properties (inscribed radius, volume etc.) recorded straight from the original voxel image. Using these realistic networks, water wet experimental data have been successfully predicted for Bentheimer [Øren et al., 1998] and Berea sandstones [Blunt et al., 2002].

[7] However, there are still remaining issues, some of which will be addressed in this paper. The approach as presented by Øren et al. [1998] assumes that lattice and subsequent flow predictions are specific to the properties of the porous medium used in the voxel-based reconstruction. We will present a methodology for combining realistic networks, originally constructed to represent one particular porous medium, with conditioning to experimental capillary pressure data to predict flow properties of a variety of different porous media.

[8] The wetting condition of the porous medium is of great importance in defining the flow characteristics. Three broad classifications can be identified: strongly water wet, strongly oil wet, or intermediate wet. A combination of these wetting conditions is also possible. We will refer to this latter condition as mixed wet; most hydrocarbon bearing reservoirs are found to fall into this category [Anderson, 1986a]. Consider that initially the rock is water-saturated and strongly water wet. Following primary oil flooding it is assumed that the part of the rock in direct contact with hydrocarbon will alter its wettability, whereas the crevices and small throats still containing water will remain strongly water wet. Initially a thin water film protects the pore walls from the asphaltenes in the oil that can make the pore walls become oil wet [Kovscek et al., 1993]. In some pores this film might collapse, allowing the pore to become oil wet. Some wettability alteration might still occur in pores where the film remains as a result of asphaltenes diffusing across the water film [Kaminsky and Radke, 1997]. The rock might therefore end up with three distinctively different simultaneous wetting populations: strongly water wet, where oil has not invaded; strongly oil wet, where the water films have collapsed; and intermediate wet, where the films remain. This type of wetting condition has indeed been observed experimentally [Masalmeh, 2003]. The same wettability state is likely to occur in groundwater situations where nonaqueous phase liquids have been in contact with the soil for many months or years.

[9] From Amott core-flood tests and nuclear magnetic resonance (NMR) it is possible to obtain a bulk indication of the wettability [Anderson, 1986b]. How the mixed wettability is distributed on the pore scale is, however, much more difficult to evaluate. Kovscek et al. [1993] proposed a theoretical model where the smaller pores become oil wet while the larger ones remain water wet. Using cryoscanning and environmental scanning electron microscopy it is possible to visualize directly the distribution of fluids at the pore scale [Fassi-Fihri et al., 1991; Combes et al., 1998; Durand and Rosenberg, 1998]. These studies suggested that the relatively simple theoretically based scenarios for mixed wetting proposed by Kovscek et al. [1993] were inadequate to described real systems, where the distribution of clay, in particular kaolinite, plays a very important role in determining what parts of the rock becomes oil wet. Only very limited mixed wet experimental relative permeability data have been predicted using pore-scale network modeling due the high degree of uncertainty relating to the characterization of mixed wettability on the pore-scale, though some promising results have been shown [Øren et al., 1998]. Jadhunandan and Morrow [1995] measured oil recovery from a series of Berea cores, where mixed wetting was achieved by varying the initial water saturation. Using network modeling the trend in recovery with wettability has been predicted successfully [Jackson et al., 2003; Oren and Bakke, 2003]. We will revisit this data set in this paper, but also investigate systems with more complex wetting behavior. The methodology for modifying networks using capillary pressure data will be combined with a new method for assigning contact angles to match measured wettability indices. A flowchart of this process is shown in Figure 1.

\section{Network Model}

[10] In this section we will outline the mathematical details and assumptions made when simulating multiphase flow in network models. This broadly follows the work of Øren et al. [1998], Patzek [2001] and Al-Futaisi and Patzek [2003]. The most basic assumption made is that the flow is capillary dominated (quasi-static) with viscous pressure drops in the model being insignificant. The capillary number $N_{c a p}$ (ratio of capillary to viscous forces) should be less than $10^{-4}$ [Blunt and Scher, 1995], as defined by

$$
N_{\text {cap }}=\frac{\mu v}{\sigma}
$$

where $\mu$ is viscosity, $\sigma$ is interfacial tension and $v$ is fluid velocity. What this means in practice is that all interfaces are kept static throughout the network with the exception of a single displacement event.

[11] We will refer to the initial wetting phase as water and the nonwetting phase as oil (unless experimental data is based on other fluids). Also, since mixed wet systems make the terms drainage (nonwetting phase invasion) and imbibition (wetting phase invasion) ambiguous, we will refer to these processes as oil and water flooding respectively.

\subsection{Description of the Pore Space}

[12] A three-dimensional voxel representation of Berea sandstone is the basis for the networks used in this paper (Figure 2). A topologically equivalent network of pores and throats is then generated with properties (radius, volume etc) extracted from the original voxel representation.

[13] The individual network elements are uniform ducts with circular, triangular or square cross-sectional shapes. These have the same shape factor [Mason and Morrow, 1991], $G=A / P^{2}$, as the voxel representation, where $A$ is the cross-sectional area and $P$ the perimeter length. In the voxel representation these are average values recorded along the throat and at the pore center. As the pore space becomes more irregular the shape factor decreases. A circle and a square have shape factors of $1 /(4 \pi)$ and $1 / 16$ respectively. For a triangle the shape factor will range from zero for a slit shaped triangle to $\sqrt{3} / 36$ for an equilateral triangle [Øren et al., 1998]. The details of how to calculate the corner half 


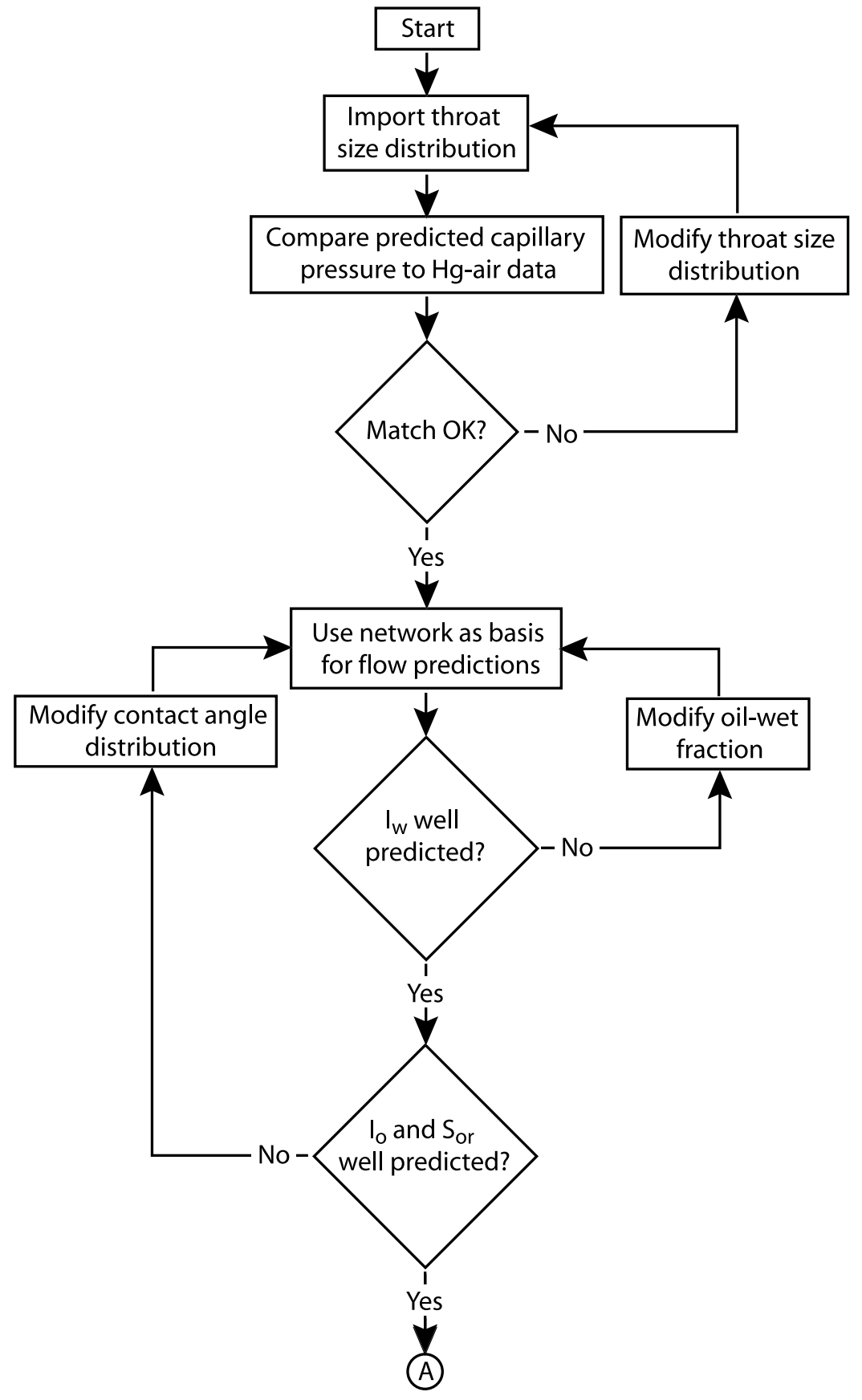

Figure 1. Flowchart for relative permeability predictions.

angles $\beta$, as illustrated in Figure 3, for a given shape factor are given in Appendix A.

[14] Using square or triangular-shaped network elements allows for the explicit modeling of wetting layers (water or oil depending on the wetting state) where nonwetting phase occupies the center of the element and wetting phase remains in the corners. The pore space in real rock is highly irregular with water remaining in grooves and crevices after primary oil flooding due to capillary forces. This behavior has been observed in micromodel experiments where flow channels typically have a square cross section [Lenormand et al., 1983]. The wetting layers might not be more than a few microns in thickness, with little effect on the overall saturation or flow. Their contribution to wetting phase connectivity is, however, of vital importance, ensuring low residual wetting phase saturation by preventing trapping.

[15] Microporosity and water saturated clays will typically not be drained during core analysis. This effect is included in the network representation by assigning a constant volume to each pore or throat that always remains water saturated.

\subsection{Primary Oil Flooding}

[16] Since all the elements (pores and throats) in the network model initially only contain water, displacement can only occur through piston-like displacement where the center of an element can only be filled if it has an adjacent element containing oil [Lenormand et al., 1983]. The cap- 

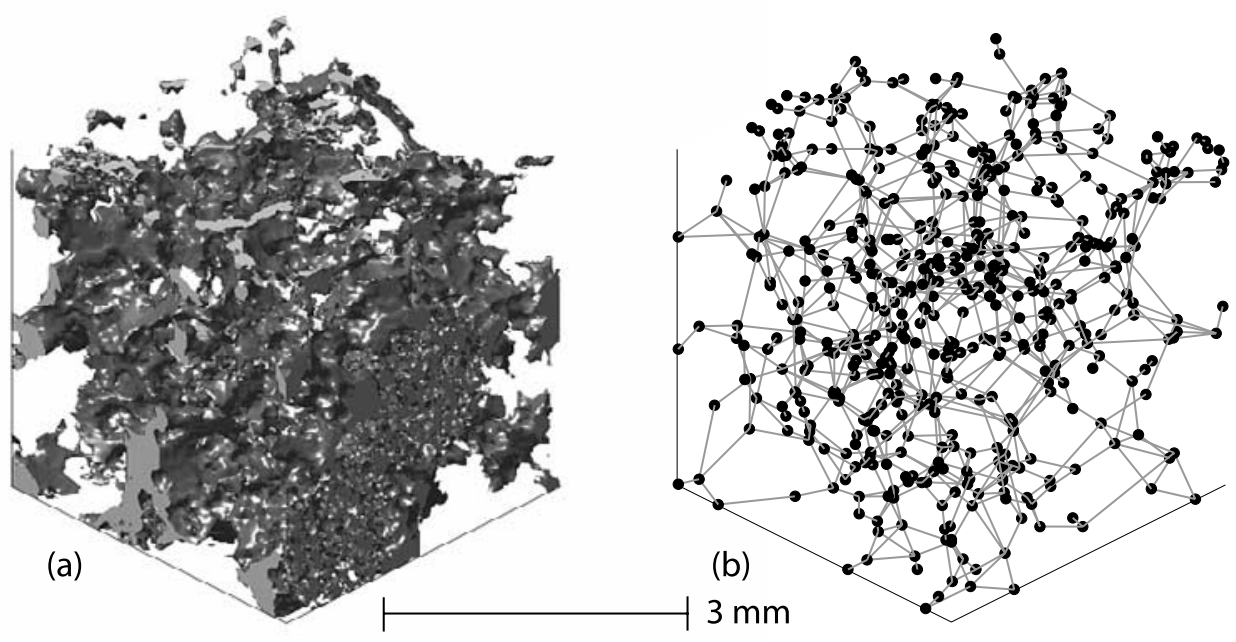

Figure 2. (a) Three-dimensional image of a sandstone along with (b) a topologically equivalent network representation [Bakke and Øren, 1997].

illary pressure required for the oil meniscus to invade a water-filled element is given by the Young-Laplace equation

$$
P_{\text {cow }}=P_{o}-P_{w}=\sigma_{o w}\left(\frac{1}{R_{1}}+\frac{1}{R_{2}}\right),
$$

where $\sigma_{o w}$ is the oil-water interfacial tension and $R_{1}$ and $R_{2}$ are the principal radii of curvature of the interface. When we know the shape of the pore and the contact angle at which the interface hits the solid surface we can use expressions that are easier to evaluate. For a circular pore it is simply

$$
P_{\text {cow }}=\frac{2 \sigma_{\text {ow }} \cos \theta_{\text {owr }}}{r},
$$

where $\theta_{\text {owr }}$ is the receding oil-water contact angle and $r$ is the inscribed radius [Dullien, 1992]. Gravity effects can be included easily by subtracting $\Delta \rho_{\text {ow }} g h$ from the capillary entry pressure expressions, where $\Delta \rho_{\text {ow }}$ is the density difference between oil and water, $g$ is the gravitational constant and $h$ is the height above datum of the element. For the remaining part of this paper we will omit the oil-water subscript.

[17] Only a very small fraction of network elements will have a circular cross section. For polygonal-shaped elements the capillary entry pressure expressions become complex as the wetting fluid remains in the corners as arc menisci (AMs). Entry pressures are found by calculating the force balance acting on the interface in the duct. This method has become known as the Mayer, Stowe and Princen (MS-P) method [Mason and Morrow, 1991] and we follow Øren et al.'s [1998] generalization of it. The details of this method are given in Appendix A.

[18] The throats along the inlet face of the network model are all assumed to be connected to a reservoir of oil. The pressure in the oil phase $P_{o}$ is then increased while the water phase pressure $P_{w}$ is kept constant throughout the network, resulting in increased capillary pressure $P_{c}$. The elements are filled in order of increasing capillary entry pressure (assuming they have an oil-filled neighbor). This process continues until some predefined saturation is reached or all elements have been filled by oil. Once a polygonal element has been filled by oil, water still remains in the corners. This will ensure that water, as the wetting phase, will remain connected throughout primary oil flooding, since escape to the outlet is always possible through wetting layers.

\subsection{Secondary Water Flooding}

[19] The part of the rock in direct contact with oil will have its wettability altered following primary oil flooding, whereas the corners and elements still only containing water will remain strongly water wet (Figure 3 ). In addition to wettability alteration, contact angles are affected by the direction of flow. Because of surface roughness, advancing contact angles $\theta_{a}$ (increase in water saturation) are typically

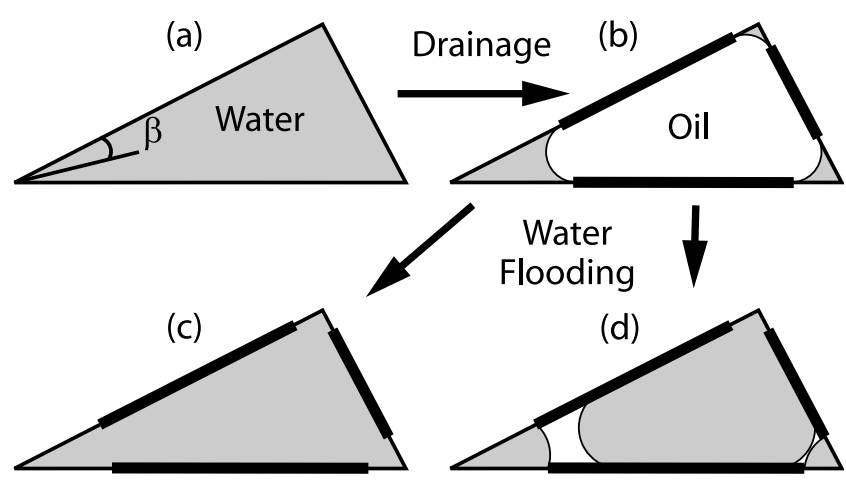

Figure 3. Possible fluid configurations. (a) Initially, the element is water-filled and strongly water wet. (b) Following primary oil flooding, the part of the element in contact with oil will alter its wettability. (c) During water flooding, the element might again become completely water-filled. (d) If wettability alteration was large enough, oil might become sandwiched as a layer between water in the corner and the center. 


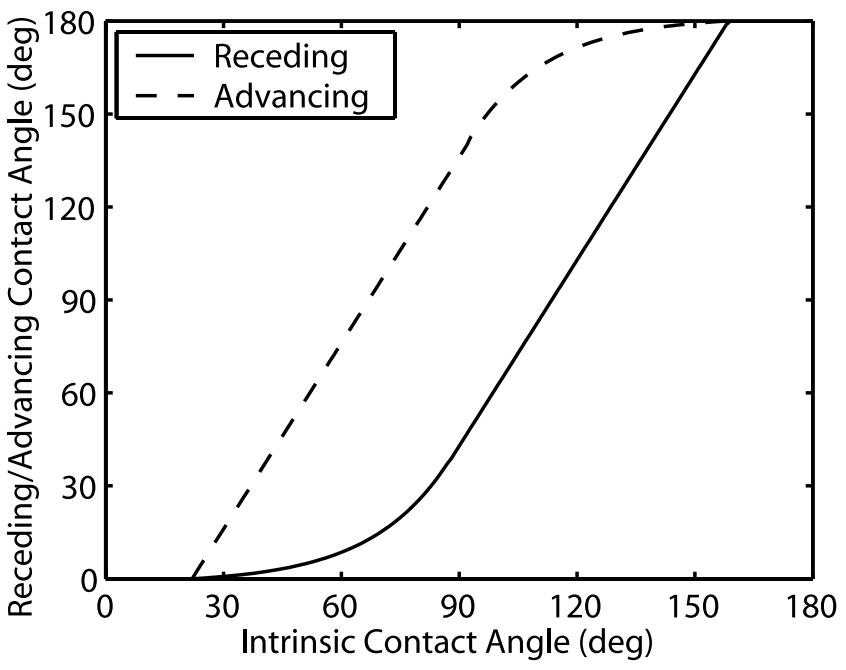

Figure 4. Relationship between receding and advancing contact angles on a rough surface as a function of intrinsic contact angle measured at rest on a smooth surface [Morrow, 1975]. We will use this relationship to find receding and advancing contact angles in our network model from a given value of intrinsic contact angle.

found to be significantly larger than receding contact angles $\theta_{r}$ (reduction in water saturation) (Figure 4) [Morrow, 1975].

[20] With wettability alteration and water in corners the mechanisms by which water can displace oil become more complex [Lenormand et al., 1983]. The three main processes are piston-like displacement, pore body filling and snap off.

\subsubsection{Piston-Like Displacement}

[21] As the capillary pressure drops, the oil-water interface in corner $i$ will initially remain pinned at the last position $b_{i}$ obtained during primary oil flooding. In order to maintain capillary equilibrium, the contact angle $\theta_{h, i}$ will hinge at this position in order to maintain capillary equilibrium.

$$
\begin{gathered}
b_{i}=R_{\min } \frac{\cos \left(\theta_{r}+\beta_{i}\right)}{\sin \beta_{i}}, \\
\theta_{h, i}=\operatorname{acos}\left(\frac{b_{i} \sin \beta_{i}}{R}\right)-\beta_{i},
\end{gathered}
$$

where $R=\sigma / P_{c}$ is the radius of curvature and $R_{\min }$ is the smallest radius obtained during primary oil flooding. Only when reaching the advancing contact angle $\theta_{a}$ will the interface start to move along the pore surface (Figure 5a). The capillary entry pressure for piston-like displacement is again found by calculating the force balance acting on the interface, with the details given in Appendix A.

\subsubsection{Pore Body Filling}

[22] The capillary entry pressure for filling a pore body during spontaneous water flooding (positive capillary pressure) is limited by the largest radius of curvature that can be achieved. This will depend on the number of adjacent oilfilled throats [Lenormand et al., 1983], as shown in Figure 6. A pore body with coordination number $z$ can thus be filled by $z-1$ possible events, $I_{1}$ to $I_{z-1}$, each occurring at a separate capillary pressure. If only a single adjacent throat is filled

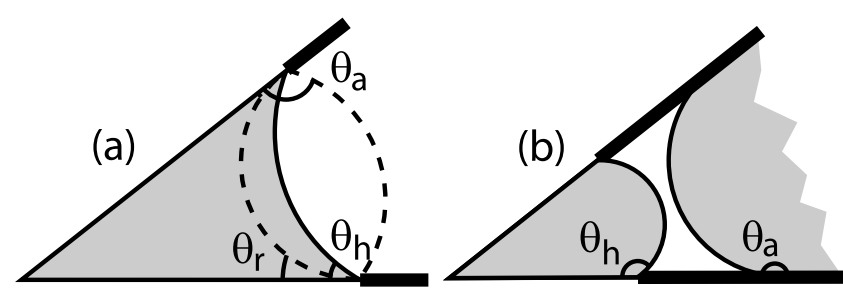

Figure 5. Fluid configurations in corners. (a) A pinned interface hinges between receding and advancing contact angles. (b) Oil might become sandwiched between water in corners and water in the center.

with oil ( $I_{1}$ mechanism) the process is similar to piston-like displacement. Since the exact spatial location of the oil-filled throats is difficult to estimate, it is common to express the capillary entry pressure as a parametric model [Blunt, 1998],

$$
P_{c}=\frac{2 \sigma \cos \theta_{a}}{r}-\sigma \sum_{i=1}^{n} A_{i} x_{i},
$$

where $n$ is the number of connecting oil-filled throats, $A_{i}$ are arbitrary numbers and $x_{i}$ are random numbers between zero and one. Since $A_{i}$ have dimensions of $\mathrm{m}^{-1}$ we chose to relate it to permeability,

$$
A_{2}-A_{n}=\frac{0.03}{\sqrt{K}}
$$

where the permeability $K$ is measured in $\mathrm{m}^{2}$. Permeability was chosen due its close relationship with the throat size distribution while allowing us to calculate the values of $A_{i}$ prior to conducting the water flooding, simplifying the estimation of capillary entry pressures. This also approximately reproduces the results by Blunt [1998], with negative capillary entry pressures possible for contact angles less than $90^{\circ}$. When only one connecting throat contains oil $\left(I_{1}\right.$ event) the process is similar to piston-like displacement and hence $A_{1}=0.0 \mu \mathrm{m}^{-1}$. This is also the most favored event. During forced water invasion (negative capillary pressure) the capillary entry pressure is not dependent on the number of adjacent oil-filled throats and the filling process is again similar to piston-like displacement.

\subsubsection{Snap Off}

[23] In snap off an element is filled with water as the result of corner water layers swelling so much that the fluid/
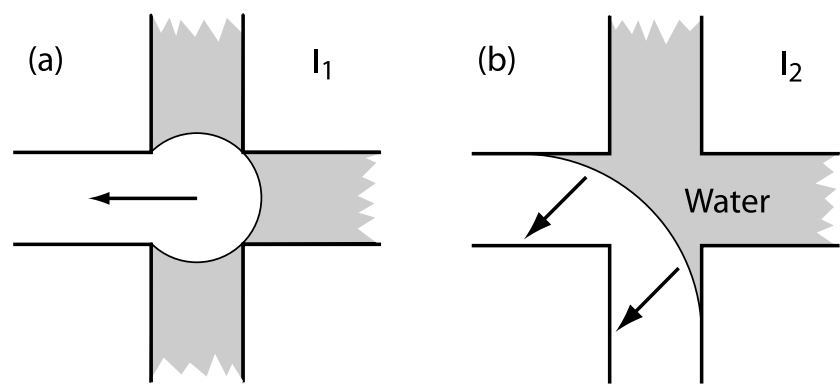

Figure 6. Pore body filling processes. The capillary entry pressure will be higher (a) when only a single throat is oilfilled than (b) when several connecting throats are filled with oil. 

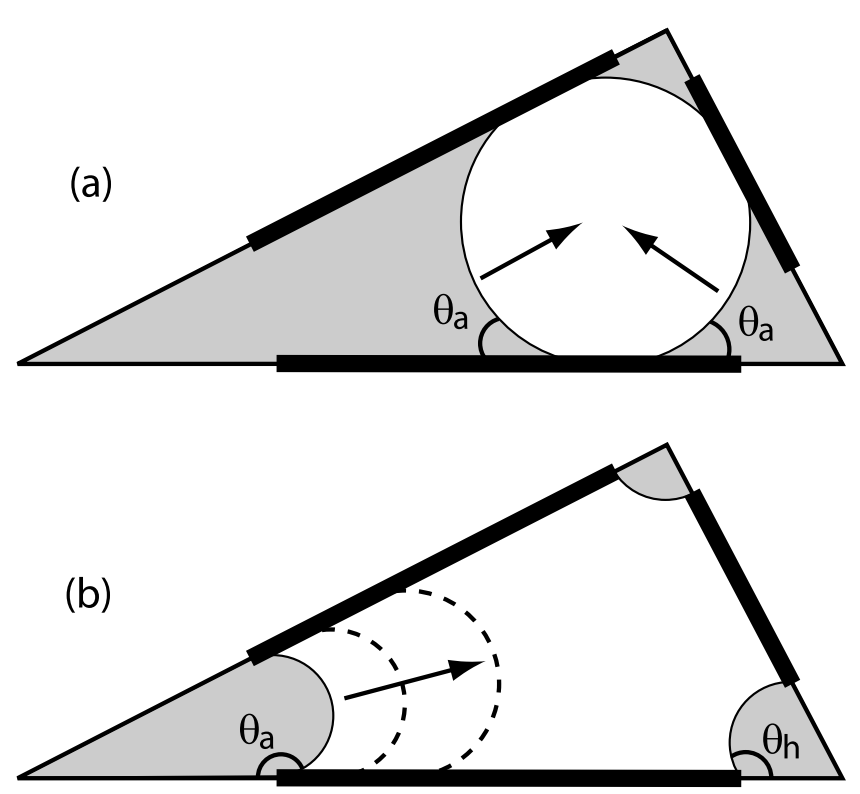

Figure 7. Snap-off events. (a) During spontaneous water injection, snap off will occur once water in corners meet along the pore wall. (b) During forced water injection, snap off will occur as soon as the advancing contact angle is reached.

fluid interface becomes unstable. Snap off will only occur if there is no adjacent element whose center is filled with water. If $\theta_{a}<\pi / 2-\beta_{1}$ the AMs will advance smoothly along the pore wall once $\theta_{a}$ has been reached. Once two AMs meet, there no longer is an oil-water-solid contact, resulting in the oil-water interface becoming unstable with spontaneous water filling as the result, as shown in Figure 7a. The capillary pressure at which this occurs depends on whether one or several AMs have started to advance along the pore wall. If two or more AMs have started to move, the AMs in the sharpest corners will meet at a capillary pressure,

$$
P_{c}=\frac{\sigma}{r}\left(\cos \theta_{a}-\frac{2 \sin \theta_{a}}{\cot \beta_{1}+\cot \beta_{2}}\right),
$$

whereas if only the AM in the sharpest corner has started to advance, it will meet the pinned AM in the most oblique corner at a capillary pressure

$$
P_{c}=\frac{\sigma}{r}\left(\frac{\cos \theta_{a} \cot \beta_{1}-\sin \theta_{a}+\cos \theta_{h, 3} \cot \beta_{3}-\sin \theta_{h, 3}}{\cot \beta_{1}+\cot \beta_{2}}\right) .
$$

The event with the highest capillary pressure will be the one that occurs.

[24] During forced water invasion snap off will occur as soon as $\theta_{h, 1}$ has reached $\theta_{a}$ or $\theta_{a}-\beta_{1}$ in the case where $\theta_{a}>$ $\pi-\beta_{1}$. Once the AM starts advancing along the pore wall, the absolute negative curvature will decrease, making it unstable with spontaneous filling as the result (Figure 7b), with the entry pressure given by

$$
P_{c}=\frac{\sigma \cos \left(\theta_{a}+\beta_{1}\right)}{R_{\min } \cos \left(\theta_{r}+\beta_{1}\right)} \quad \theta_{a} \leq \pi-\beta_{1}
$$

or

$$
P_{c}=\frac{-\sigma}{R_{\min } \cos \left(\theta_{r}+\beta_{1}\right)} \quad \theta_{a}>\pi-\beta_{1} .
$$

[25] Piston-like displacement is always favored (occurring at a higher capillary pressure) over snap off wherever piston-like displacement is topologically possible. Snap off can, however, still be an extremely important displacement process as it does not require any adjacent elements to be water-filled, hence it can occur anywhere where the oil is connected to the outlet.

\subsubsection{Oil Layers}

[26] When the water interface enters the element during forced invasion, a layer of oil (Figure 5b) might become sandwiched between water in the corner and that in the center if

$$
\theta_{a}>\frac{\pi}{2}+\beta_{i}
$$

This can occur because the inner oil-water interface has a hinging contact angle that might be much less than the advancing contact angle associated with the outer interface. Stable oil layers can significantly increase recovery as it maintains oil connectivity in much the same way that water layers influence primary oil flooding. The oil layer will collapse once the two interfaces meet, which if the capillary pressure is the same across the two interfaces, is given by

$$
P_{c}=\frac{\sigma \cos \left(\operatorname{acos}\left(2 \sin \beta_{i}+\cos \theta_{a}\right)+\beta_{i}\right)}{b_{i} \sin \beta_{i}}
$$

\subsubsection{Displacement Process}

[27] At the beginning of water flooding the reservoir is at some given initial water saturation and capillary pressure. Water is then injected by increasing the water phase pressure, keeping the oil phase pressure constant through the outlet reservoir, resulting in a reduced capillary pressure. All possible displacement events are sorted in terms of capillary entry pressure, with the event with the highest capillary pressure executed first. The initial list of possible events is much bigger than it was during oil flooding. Not only are elements with adjacent water included, but also all possible snap-off events.

[28] During primary oil flooding trapping was unimportant (though possible because oil-filled elements with a circular cross section do not contain water layers) as water in corners ensured global connectivity, whereas during water flooding it becomes crucial. Once an element is filled from spontaneous invasion, it no longer conducts oil, since it is completely water-filled. Snap-off events, which typically occur in small throats, will reduce oil connectivity even further. Once a cluster of oil-filled elements becomes trapped it no longer is in pressure communication with the outlet. The capillary pressure within that cluster is frozen and the oil within it cannot be displaced. If the model is primarily oil wet, trapping again becomes less important as the oil can escape through oil layers.

\subsection{Subsequent Flooding Cycles}

[29] We assume that no further wettability alteration takes place following that occurring after primary oil flooding. 
Secondary oil flooding might thus become a spontaneous process. The possible displacement events occurring are the same as those described for water flooding. If oil layers are present we assume that the capillary entry pressure is only affected by the outer oil-water interface, which initially is pinned at the last position attained during water flooding. The entry pressure is calculated analogously to spontaneous piston-like water invasion. Oil snap off is also possible as long as water and oil is not trapped.

[30] If secondary water flooding progressed down to residual oil saturation, most oil layers will typically have collapsed, though we assume a molecular film of oil will remain. As the capillary pressure increases during secondary oil flooding these layers will reform if a neighboring element contains nontrapped oil either in layers or in the center. The capillary pressure at which this happens will depend on whether any of the water, either in the corners or in the center, is trapped. If none is trapped the capillary pressure is given by equation (13) and we assume that the outer interface will subsequently hinge at the last position before the layer collapsed. Expressions for cases where the water is trapped are given in Appendix A along with expressions where both interfaces are hinging, as might be the case in higher-order water flooding cycles.

\subsection{Calculation of Transport Properties}

[31] Estimation of transport properties can be done at any point during the displacement. Saturation is calculated using the prevailing maximum in capillary pressure (or minimum in the case of water injection) to compute the radius of curvature of the fluid interfaces. It is assumed that fluid flow in each phase is independent of the other phase and that the network configuration, i.e. the location of all the fluid interfaces is frozen in place. The absolute permeability $K$ of the network is found from Darcy's law,

$$
K=\frac{\mu_{p} q_{\text {tsp }} L}{A\left(\Phi_{\text {inlet }}-\Phi_{\text {outlet }}\right)},
$$

when the network is fully saturated with a single-phase $p$ of viscosity $\mu_{p}$. The total single-phase flow rate $q_{t s p}$ through the network is found by imposing a potential drop $\left(\Phi_{\text {inlet }}-\right.$ $\Phi_{\text {outlet }}$ ) across its length $L$, with $A$ being the cross-sectional area of the model. Potential is defined as $\Phi=P-\rho_{p} g h$. The relative permeability $k_{r p}$ is then given by

$$
k_{r p}=\frac{q_{\mathrm{tmp}}}{q_{\text {tsp }}},
$$

where $q_{\text {tmp }}$ is the total flow rate of phase $p$ in multiphase conditions with the same imposed pressure drop. The total flow rate is found by solving for the pressure everywhere, imposing mass conservation at every pore $i$,

$$
\sum_{\mathrm{j}} q_{p, i j}=0,
$$

where $j$ runs over all the throats connected to pore $i$. For this to be valid we must assume the flow to be incompressible and that the pressure drops due to flow are insignificant compared to the capillary pressure. This is consistent with the previously stated assumptions about capillary dom- inance. The flow rate $q_{p}$ between two pores $i$ and $j$ is given by

$$
q_{p, i j}=\frac{g_{p, i j}}{L_{i j}}\left(\Phi_{p, i}-\Phi_{p, j}\right),
$$

where $g_{p}$ is the fluid conductance, $L$ is the length between the pore centers and $\Phi_{p}$ is the phase potential. The conductance between two pore bodies $g_{p, i j}$ is taken to be the harmonic mean of each individual conductance,

$$
\frac{L_{i j}}{g_{p, i j}}=\frac{L_{i}}{g_{p, i}}+\frac{L_{t}}{g_{p, t}}+\frac{L_{j}}{g_{p, j}},
$$

where $t$ indicates the connecting throat. The pore body lengths, $L_{i}$ and $L_{j}$, are the lengths from the pore-throat interface to the pore center. A linear set of equations can be defined from equations (16) and (17) that can be solved in terms of pore pressures. With the pressures known at either side of any cross-sectional plane within the network model, the total flow rate can be computed from equation (17). To prevent boundary effects influencing relative permeability predictions, only the last half of the network is used for these calculations.

[32] For single-phase laminar flow in a circular tube the conductance $g_{p}$ is given analytically by Poiseuille's law,

$$
g_{p}=k \frac{A^{2} G}{\mu_{p}}=\frac{1}{2} \frac{A^{2} G}{\mu_{p}} .
$$

Analytical expressions for equilateral triangles and squares can also be developed [Patzek and Silin, 2001] with $k$ being $3 / 5$ and 0.5623 respectively. From numerical simulations, Øren et al. [1998] found that the conductance for an irregular triangle was closely approximated by equation (19), using the same constant $k$ as for an equilateral triangle, $3 / 5$.

[33] In multiphase conditions an individual polygonal network element might contain both oil and water. Expressions for flow in element centers (where the corners contain a different phase) and flow in water and oil layers are all needed. These expressions are typically derived empirically from numerical simulations of flow in arbitrarily shaped ducts. For flow in the center we continue to use equation (19) but multiply it by the fraction occupied by the center, whereas flow in layers require separate expressions as the geometry becomes more complex. These are given in Appendix A.

\section{Predicting Water Wet Experimental Data}

[34] To validate the model we need to compare against experimental results. In this section we will be using a network generated from reconstructed Berea sandstone [Lerdahl et al., 2000] to predict a wide range of experimental data conducted on several types of porous media. The network covers a rock volume of $3^{3} \mathrm{~mm}^{3}$, consisting of 12,349 pores and 26,146 throats. The connection numbers varies between 1 and 19, with an average of 4.19. The porosity of the network is 0.24 with an absolute permeability of 2.5 Darcy.

[35] In this section we will focus on water wet experimental data where the pore-level wettability is fairly easily 

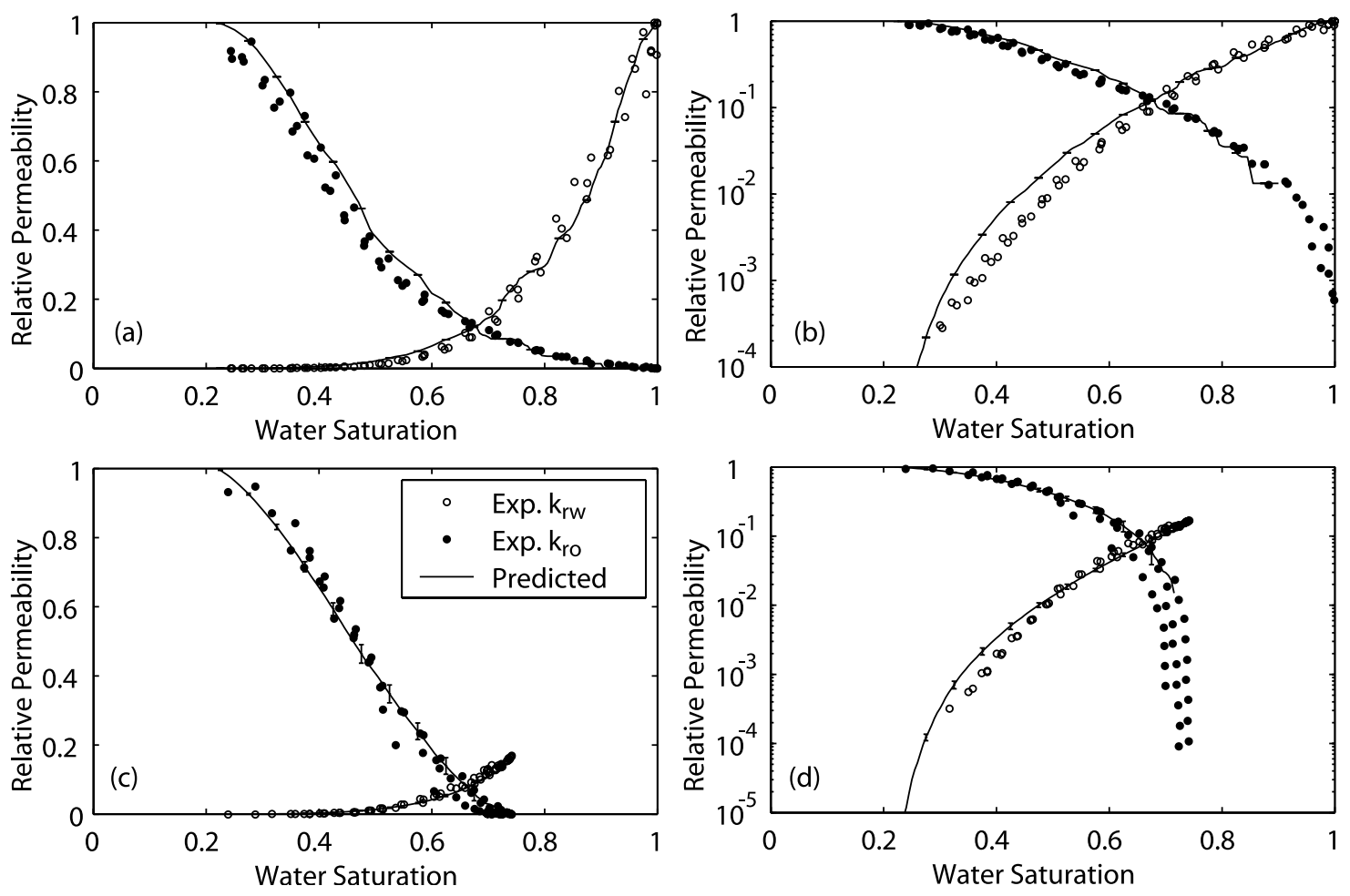

Figure 8. ( $a-b)$ Predicted primary oil flooding and $(c-d)$ secondary water flooding relative permeability for water wet Berea sandstone (lines) compared to experimental data by Oak [1990] (circles). The predicted results are the mean of 20 realizations, and the length of the error bars are twice the standard deviation. Plotted on linear scale in Figures $8 \mathrm{a}$ and $8 \mathrm{c}$ and on semilogarithmic scale in Figures $8 \mathrm{~b}$ and $8 \mathrm{~d}$.

characterized. We will start with data from Berea sandstones, where we know we have an appropriate network, allowing us to validate the basic network model. Subsequently we will investigate flow in sand packs. This poses a challenge since we have to modify our network to be adequately representative of the porous medium.

[36] As pore-scale network modeling has grown in complexity the amount of possible input parameters has grown. However, for this work the only parameters we will modify are the networks, through a well-defined process, and the pore-level wetting state, defined by the intrinsic contact angle $\theta_{i}$. All other parameters are kept fixed for all predictions. Most of the experimental data we analyze are based on steady state measurements. Unsteady state data, though more abundant, require additional numerical simulations to calculate relative permeability, increasing experimental uncertainty.

\subsection{Berea Sandstone}

[37] Predicted relative permeability for primary oil flooding and water flooding are compared to steady state experimental data by Oak [1990] in Figure 8. During primary oil flooding the network is assumed to be strongly water wet with a receding contact angle of $0^{\circ}$. There are no other parameters to adjust, with all geometric network properties (connection numbers, radii, shape factors etc.) defined in the sandstone reconstruction process. While the network is still water wet during water flooding the advancing contact angles will be larger, due to roughness of the surface and minor wettability alteration. The prediction shown in
Figures $8 \mathrm{c}$ and $8 \mathrm{~d}$ was obtained with intrinsic contact angles uniformly distributed between $50^{\circ}$ and $62^{\circ}$. The corresponding advancing contact angles can be found from Figure 4. Small changes in the distribution of contact angles did not significantly affect the results, as long as the system remained water wet. Additional fluid and rock parameters are given in Table 1. The predictions are the mean of 20 realizations with the length of the error bars being twice the standard deviation. The network topology and the pore and throat sizes are the same for all realizations. The statistical variation is mainly the result of contact angles being randomly distributed. During primary oil flooding there is hardly any variation as contact angles are kept at a constant $0^{\circ}$, though there is some small variation in the network due to the random assignment of the intermediate corner half angle.

\subsection{Sand Pack}

[38] When using pore-scale modeling to predict experimental data it is clearly important that the underlying

Table 1. Fluid and Rock Properties Used in Predictions of Experimental Data by Oak [1990]

\begin{tabular}{lc}
\hline \multicolumn{1}{c}{ Property } & Value \\
\hline Connate water saturation & 0.22 \\
Interfacial tension $\sigma, 10^{-3} \mathrm{~N} / \mathrm{m}$ & 30.0 \\
Water viscosity $\mu_{w}, 10^{-3} \mathrm{~Pa} / \mathrm{s}$ & 1.05 \\
Oil viscosity $\mu_{o}, 10^{-3} \mathrm{~Pa} / \mathrm{s}$ & 1.39 \\
\hline
\end{tabular}



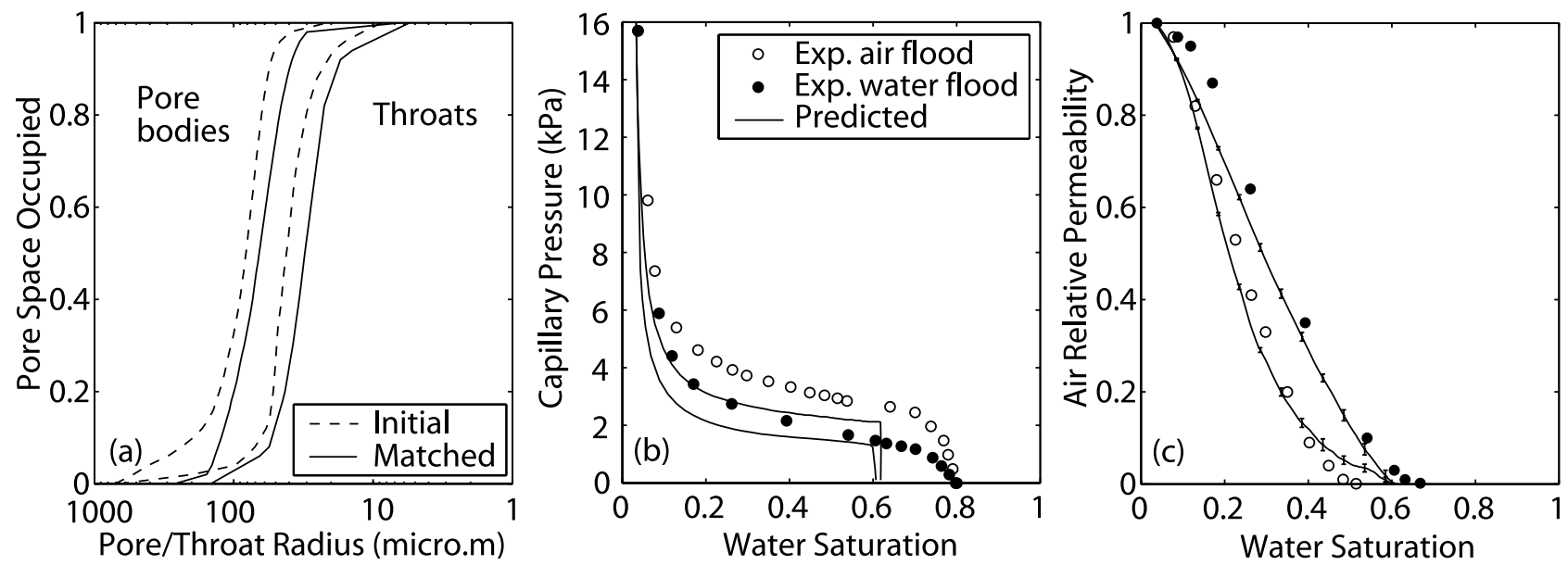

Figure 9. Comparison between predicted properties and experimental data by Dury et al. [1998]. (a) Using the experimental capillary pressure and a bundle of capillary tubes, an initial estimate for the throat size distribution can be made. However, the predictions for (b) capillary pressure and (c) relative permeability are poor, indicating that the pore size distribution needs to be adjusted to match the data.

network is representative of the rock. However, if the exact rock type has to be used for the network construction the application of predictive pore-scale modeling will be severely limited due to the complexity and cost of methods such as X-ray microtomography. In this section we will use the topological information of the Berea network (relative pore locations and connection numbers) while tuning the properties of the individual network elements using capillary pressure data. This modified network will be used to predict the flow properties of a sand pack as measured by Dury [1997] and Dury et al. [1998]. The experimental data are based on air-water flow with capillary pressure and air relative permeability available for secondary air flooding and tertiary water flooding, also referred to as the main flooding cycles. The relative permeabilities were obtained using the stationary liquid method. In this case air is the nonwetting phase, and is treated identically to oil.

[39] The capillary pressures for the sand pack are shown in Figure 9. By assuming the network to be a bundle of capillary tubes it is possible to obtain an initial estimate of the throat size distribution using the air flooding capillary pressures and equation (3). Intrinsic contact angles used in the network model are consistent with experimentally measured values (Table 2) and during air flooding the model is strongly water wet. From Figure 9 it is, however, clear that the predicted capillary pressure is not close to the experimental data. This indicates the difficulty of predicting multiphase data: the capillary pressure and relative permeabilities are influenced by the spatial distribution of pores and throats and their connectivity, something that cannot be captured using a bundle of capillary tubes assumption. The distribution of throat sizes is subsequently modified iteratively until an adequate match is obtained against the experimental air flooding data (Figure 10), with individual network elements assigned inscribed radii from the target distribution while still preserving their rank order; that is, the largest throat in the network is given the largest radius from the target distribution and so on. This should ensure that size correlations between individual elements and on larger scales are maintained. Modifications to the throat size distribution at each iteration step were done by hand rather than by any optimization technique. The results are insensitive to the details of how the throat sizes are adjusted.

[40] Capillary pressure hysteresis is a function of both the contrast between pore body and throat radii and the contact angle hysteresis. The radii of the pore bodies is determined from

$$
r_{p}=\max \left(\alpha \frac{\sum_{i=1}^{n} r_{i}}{n}, \max \left(r_{i}\right)\right),
$$

where $n$ is the connection number and $\alpha$ is the aspect ratio between the pore body radius $r_{p}$ and connecting throat radii $r_{i}$. The grain size distribution in a sand pack is typically more uniform than that found in a Berea sandstone, resulting in reduced size contrast between pore bodies and throats. The maximum value in the original Berea network, which is close to 50 , is thus unlikely to be found in a network representing a sand pack. A good match to experimental water flood capillary pressure is achieved by distributing the aspect ratios between 1.0 and 5.0 with a mean of 2.0. This distribution is very similar to that of the original Berea network, though with a lower maximum value. The absolute size of the model, defining individual pore and throat lengths, is adjusted such that the average ratio of throat length to radius is maintained from the original network. Pore and throat volumes were adjusted such that the target

Table 2. Fluid Properties Used in Predictions of Experimental Data by Dury et al. [1998]

\begin{tabular}{lll}
\hline & Water & Air \\
\hline Interfacial tension $\sigma, 10^{-3} \mathrm{~N} / \mathrm{m}$ & 70.25 & - \\
Measured advancing contact angle $\theta_{a}{ }^{\mathrm{a}}{ }^{\mathrm{a}} \mathrm{deg}$ & 19.9 & - \\
Measured receding contact angle $\theta_{r}{ }^{\mathrm{a}} \mathrm{deg}$ & 13.5 & - \\
Intrinsic contact angles used $\theta_{i}$, deg & $30-40$ & - \\
Density $\rho, \mathrm{kg} / \mathrm{m}^{3}$ & 1000 & 1.22 \\
Viscosity $\mu, 10^{-3} \mathrm{~Pa} / \mathrm{s}$ & 0.97 & 0.018 \\
\hline
\end{tabular}

${ }^{\mathrm{a}}$ Measured using Wilhelmy plate method. 

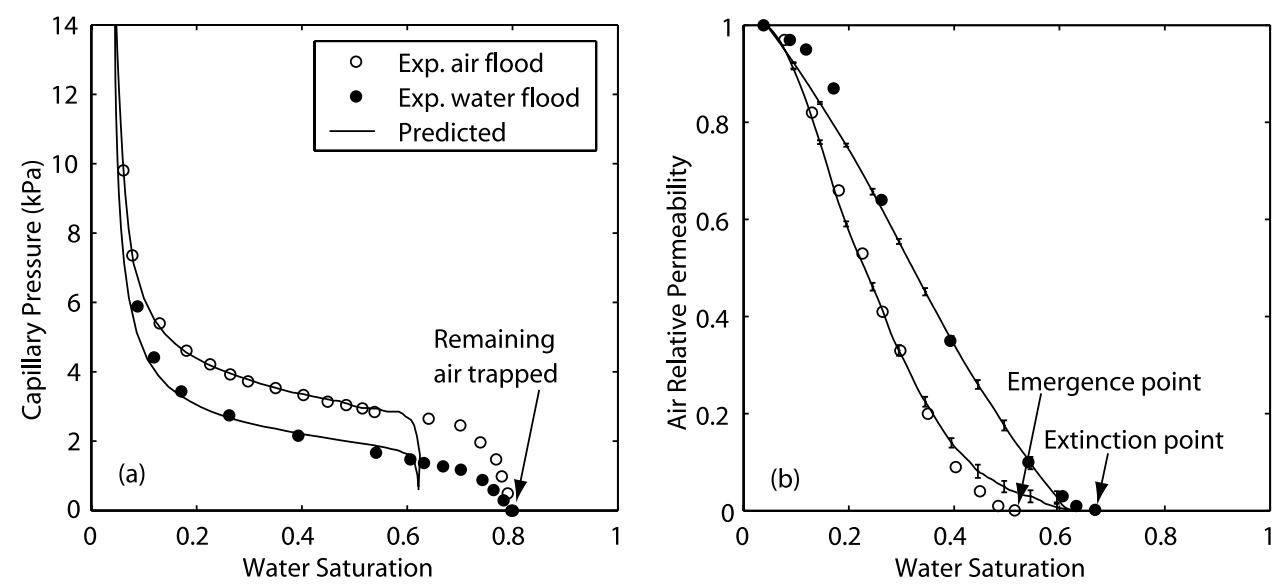

Figure 10. Comparison between predicted properties and experimental data by Dury et al. [1998] following a network modification process to match the capillary pressure data. (a) The match to experimental capillary pressure is excellent, except at high water saturations. (b) Predictions of air relative permeability are also improved.

porosity was achieved, again maintaining the rank order. The final pore body and throat size distributions are shown in Figure 9. Both distributions are shifted toward smaller values compared to the initial estimates.

[41] In Figure 10 the predicted air relative permeability for secondary air flooding and tertiary water flooding are compared to experimental data by Dury et al. [1998]. For experimental convenience the relative permeability was calculated using the air flow rate at connate water saturation $S_{w c}$ rather than that at single phase conditions as defined by equation (15). This has, however, little influence on the results since our computed air relative permeability is 0.99 at $S_{w c}$. Both the relative permeability hysteresis and the extinction point (where air no longer flows across the model) following water flooding are well predicted.

[42] From the capillary pressure curve we appear to predict much higher residual gas saturation than in the experiments. Dury [1997] suggested that the large difference in saturation between where all remaining air is trapped and where it is able to flow across the pack could be partly related to air compressibility but more likely is due to the displacement of clusters of air not spanning the network. In the network model this difference is in the order of a few percentage points, rather than the almost $20 \%$ seen in the experiments.

[43] The large experimental difference between the emergence point (where air is first able to flow across the model during air flooding) and the extinction point, something that is not observed in the network model, is difficult to explain physically. Using a network originally constructed to represent a consolidated Berea sandstone, it is possible that the contrast in size between pore bodies and connecting throats is still overpredicted, resulting in snap off becoming too dominant. However, this is unlikely to explain why the network model predicts considerably smaller difference between the emergence and extinction points. With snap off being less dominant, the number of trapped clusters following water flooding should be smaller. It would be natural to expect that fewer forced displacement events are needed before a gas cluster once again spans the network. Hence an overprediction of the size contrast between pore bodies and throats should, if anything, lead to an over- prediction of the difference between emergence and extinction points.

[44] The methodology of tuning the properties of the network to experimental capillary pressure, which is fairly easy to obtain, in order to match transport properties such as relative permeability, which undoubtedly is more difficult to obtain, has been attempted by several authors [Wise, 1992; Rajaram et al., 1997; Fischer and Celia, 1999]. What distinguishes this method is that we use networks that are constructed directly from realistic images of porous media. Our assumption is that the higher-order topological information, which Sok et al. [2002] found was crucially important if reliable predictions of transport properties were to be made, is less specific to a given system than are properties such as pore size distribution. By preserving the rank order of the individual network elements we not only maintain topological information but we also go some way toward resolving the problem of nonuniqueness as noted by Vogel [2000]. Fischer and Celia [1999] also attempted to predict this data [Dury et al., 1998]. Although the relative permeability for the water flooding cycle was reasonably well predicted, they were unable to predict the observed hysteresis, resulting in a poor prediction for the gas flooding cycle. With the approach presented here we have been able to successfully predict relative permeability for both flooding cycles.

\section{Wettability Effects on Experimental Data}

[45] Most hydrocarbon-bearing reservoir rocks are found to be mixed wet, where parts of the grain surface have become oil wet while the rest remains water wet. We will first predict data from mixed wet Berea cores. Though the network geometry should still be valid, pore-level wettability and residual saturations are more uncertain. We will subsequently try to predict mixed wet relative permeability for other rock types. This poses a challenge since we have to modify our network while at the same time try to characterize the pore-level wetting state.

[46] The wetting states of the cores are typically determined using the Amott method [Anderson, 1986b], mea- 
Table 3. Fluid Properties Used in Predictions of Experimental Data by Jadhunandan and Morrow [1995]

\begin{tabular}{lc}
\hline \multicolumn{1}{c}{ Property } & Value \\
\hline Interfacial tension $\sigma, 10^{-3} \mathrm{~N} / \mathrm{m}$ & 12.0 \\
Water viscosity $\mu_{w}, 10^{-3} \mathrm{~Pa} / \mathrm{s}$ & 0.99 \\
Oil viscosity $\mu_{o}, 10^{-3} \mathrm{~Pa} / \mathrm{s}$ & 5.23 \\
\hline
\end{tabular}

suring the fraction of each phase $p$ that spontaneously imbibes,

$$
I_{p}=\frac{\Delta S_{p s}}{\Delta S_{p s}+\Delta S_{p f}}
$$

where $\Delta S_{p s}$ and $\Delta S_{p f}$ are the spontaneous and forced parts of the displacement. It is typical to report the wetting state using a single Amott-Harvey index $I_{w-o}=I_{w}-I_{o}$, where $I_{w}$ and $I_{o}$ are the water and oil indices.

\subsection{Mixed Wet Berea Sandstone}

[47] The influence of wettability on oil recovery during water flooding has been extensively investigated by Jadhunandan and Morrow [1995]. Experiments were conducted on 50 Berea core samples. Following primary oil flooding to some initial water saturation, between 7.9 and $32 \%$, the cores were aged, during which the wettability was altered, and then subsequently water flooded until 20 pore volumes had been injected.

[48] Using the network model combined with BuckleyLeverett analysis [Dullien, 1992] the experiments were reproduced to see if the same trends and results could be predicted. All cores were assumed to have connate water saturation $S_{w c}$ of $7.9 \%$, equal to the lowest initial saturation in the experiments. Following primary oil flooding to some initial water saturation $S_{w i}$, all network elements contacted by oil were assumed to have their wettability altered. Oil recovery was determined from the secondary water flooding cycle. Finally the Amott wettability indices were determined with additional oil and water flooding cycles. All cycles except primary oil flooding were terminated follow- ing 20 pore volumes injected with the corresponding residual saturation and oil recoveries determined from a one-dimensional Buckley-Leverett analysis using the predicted network relative permeability and ignoring capillary pressure. Fluid parameters are given in Table 3.

[49] Careful attention to wettability characterization and displacement processes is needed to explain the surprising experimental results shown in Figure 11a, where as $S_{w i}$ increases recovery initially increases but then decreases again for the largest values. The simplest scenario is that for a given crude oil, brine and rock condition the distribution of contact angles in pores contacted by oil during primary oil flooding is similar regardless of initial water saturation [Jackson et al., 2003]. The wettability variation observed through the Amott indices is then just a function of $S_{w i}$, with the rock becoming less oil wet as $S_{w i}$ increases since fewer pores have their wettability altered. By matching the observed wettability indices at a given initial water saturation it should then be possible to predict the trends in recovery and wettability as $S_{w i}$ is varied. With connate water saturation chosen as the reference case the wetting state was reasonably well matched with intrinsic contact angles in wettabilityaltered pores uniformly distributed between $85^{\circ}$ and $120^{\circ}$. The Amott water $I_{w}$ and oil $I_{o}$ indices were calculated to be 0.00 and 0.47 , the same as the experimental values.

[50] Oil recovery is a direct function of the fractional water flow $f_{w}$, which in the one-dimensional BuckleyLeverett analysis is given by,

$$
f_{w}\left(S_{w}\right)=\frac{1}{1+\frac{k_{r o}\left(S_{w}\right) \mu_{w}}{k_{r w}\left(S_{w}\right) \mu_{o}}} .
$$

From equation (22) it is clear that a low water and high oil relative permeability is advantageous to oil recovery. Relative permeability curves for different $S_{w i}$ are shown in Figure 12, along with water fractional flow curves and capillary pressures. Although snap off is possible, it is highly unfavorable compared to piston-like displacement in oil wet conditions. Hence water will typically enter the model through piston-like displacement from existing
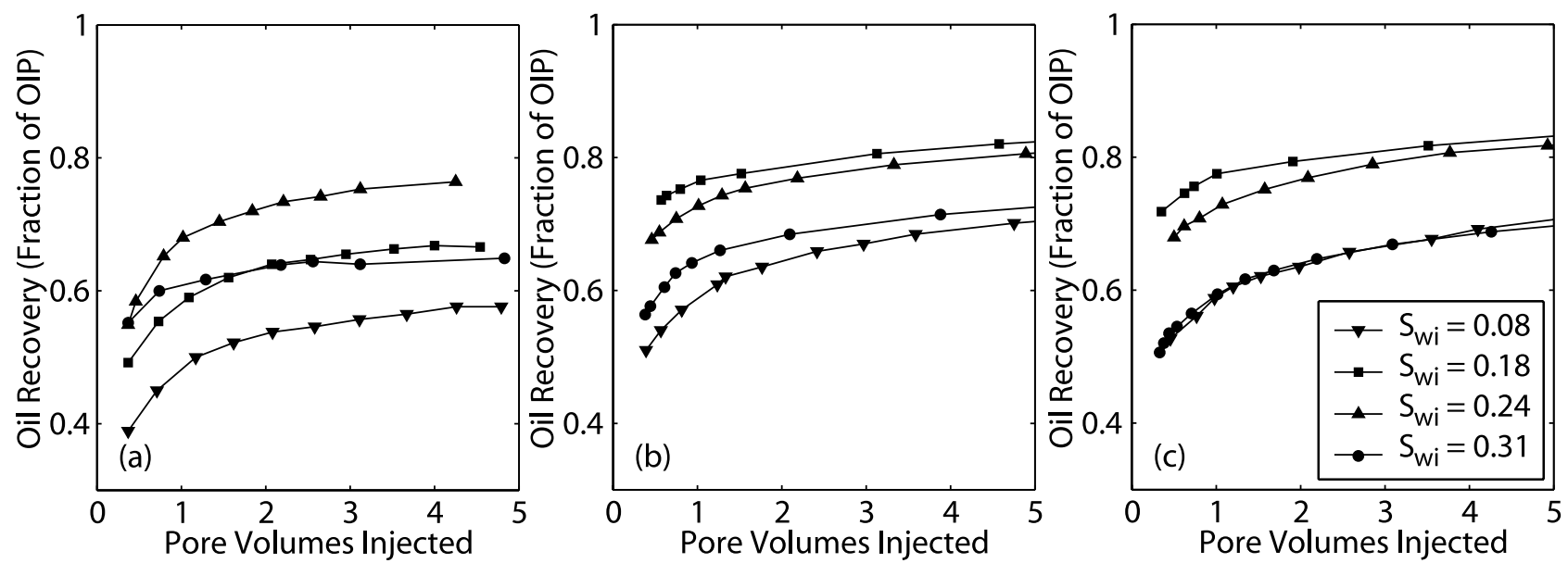

Figure 11. Oil recovery for different initial water saturations. (a) Experimental data from Jadhunandan and Morrow [1995]. (b) Predicted recoveries using pore-scale modeling with a fixed distribution of intrinsic contact angles between $85^{\circ}$ and $120^{\circ}$. (c) Predictions where fewer pores become oil wet for $S_{w i}$ above 0.20 . 

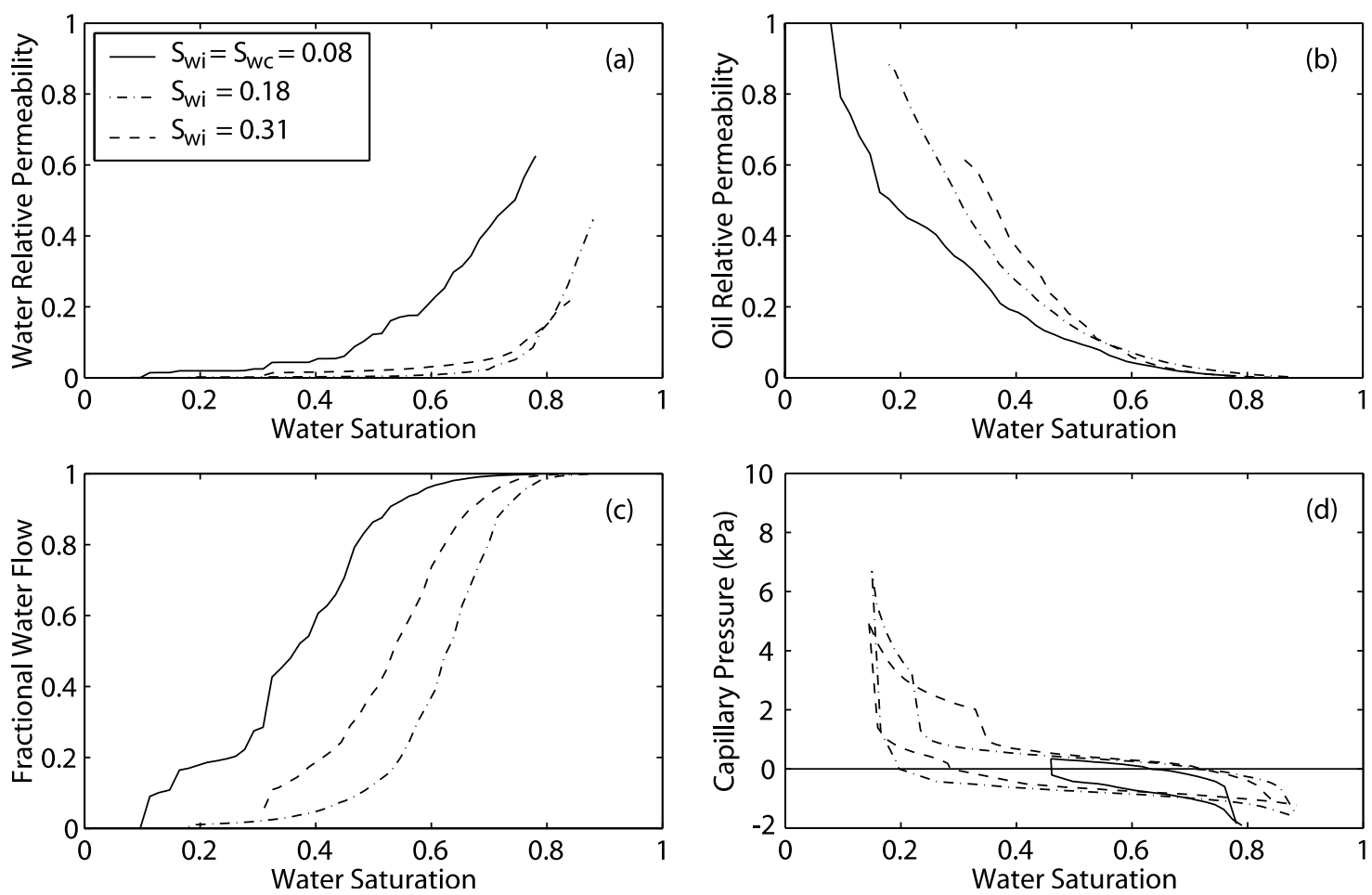

Figure 12. Influence of $S_{w i}$ on macroscopic flow properties during secondary water flooding. We have assumed that all pores contacted by oil after primary oil flooding have a fixed distribution of contact angles between $85^{\circ}$ and $120^{\circ}$, corresponding to the predictions shown in Figure $11 \mathrm{~b}$. Each flooding cycle is terminated after 20 pore volumes have been injected. (a) Water relative permeability. (b) Oil relative permeability. (c) Fractional water flow. (d) Capillary pressure during secondary oil flooding and tertiary water flooding.

water-filled elements. If $S_{w i}$ is low there are relatively few of these left following primary oil flooding, resulting in water entering the model though large well-connected clusters. As $S_{w i}$ increases, the number of initially water-filled elements will increase rapidly. These are typically isolated elements, resulting in water entering the system through many clusters only connected through layers. This behavior has significant impact on relative permeability since flow conductance in layers is typically orders of magnitude less than that in pore centers. In cases where there are many initial clusters, water has to repeatedly flow through layers to connect from inlet to outlet, resulting in low relative permeability, as evident from the $S_{w i}=0.18$ case in Figure 12. Since the initially oilfilled elements are oil wet, water preferentially fills the larger pores and throats. This results in a large increase in water saturation with little change in water connectivity and hence water relative permeability. Only at high water saturations, around 0.7 , will the water clusters start to coalesce, resulting in a rapid increase in water relative permeability. In the limiting case where $S_{w i}=S_{w c}$ there is only a single water cluster originating from the inlet reservoir, which rapidly establishes a connected path of water-filled elements across the network giving a more gradual increase in $k_{r w}$.

[51] There is less effect of $S_{w i}$ on the oil relative permeability. The last elements to be filled during primary oil flooding are the smallest ones that also contribute the least to oil conductance, whereas the first elements filled during water flooding in oil wet conditions are the largest, resulting in large oil relative permeability hysteresis. The oil relative permeability for a given $S_{w i}$ will thus lie above those starting from a lower $S_{w i}$.

[52] The effects on both oil and water relative permeability combines to give lower fractional water flow and subsequently higher recovery as $S_{w i}$ increases. There is, however, an optimal $S_{w i}$ above which recovery again starts to decrease, as evident from Figures 11 and 12c. Elements filled by water during water flooding still conduct oil due to oil layers, which is not the case for elements that remain water-filled from primary oil flooding. This results in increased oil trapping with $S_{w i}$, reducing oil relative permeability. Also, at some point so many elements still contain water at the beginning of water flooding that large water clusters can easily form, increasing the water relative permeability. Whereas relative permeability and fractional flow show a nonuniform trend with $S_{w i}$, this is not the case for the Amott wettability indices (Table 4), calculated from the capillary pressures as shown in Figure 12.

Table 4. Variation in Amott Wettability Indices for Different Initial Water Saturations

\begin{tabular}{|c|c|c|c|c|c|c|}
\hline \multirow[b]{2}{*}{$S_{w i}$} & \multicolumn{2}{|c|}{$\begin{array}{c}\text { Experimental } \\
\text { Data }\end{array}$} & \multicolumn{2}{|c|}{$\begin{array}{c}\text { Fixed } \\
\text { Wettability }\end{array}$} & \multicolumn{2}{|c|}{$\begin{array}{c}\text { Variable } \\
\text { Wettability }\end{array}$} \\
\hline & $I_{w}$ & $I_{o}$ & $I_{w}$ & $I_{o}$ & $I_{w}$ & $I_{o}$ \\
\hline 0.079 & 0.00 & 0.47 & 0.00 & 0.47 & 0.00 & 0.47 \\
\hline 0.180 & 0.02 & 0.20 & 0.06 & 0.23 & 0.03 & 0.19 \\
\hline 0.240 & 0.20 & 0.07 & 0.13 & 0.21 & 0.20 & 0.06 \\
\hline 0.311 & 0.34 & 0.00 & 0.19 & 0.18 & 0.34 & 0.01 \\
\hline
\end{tabular}




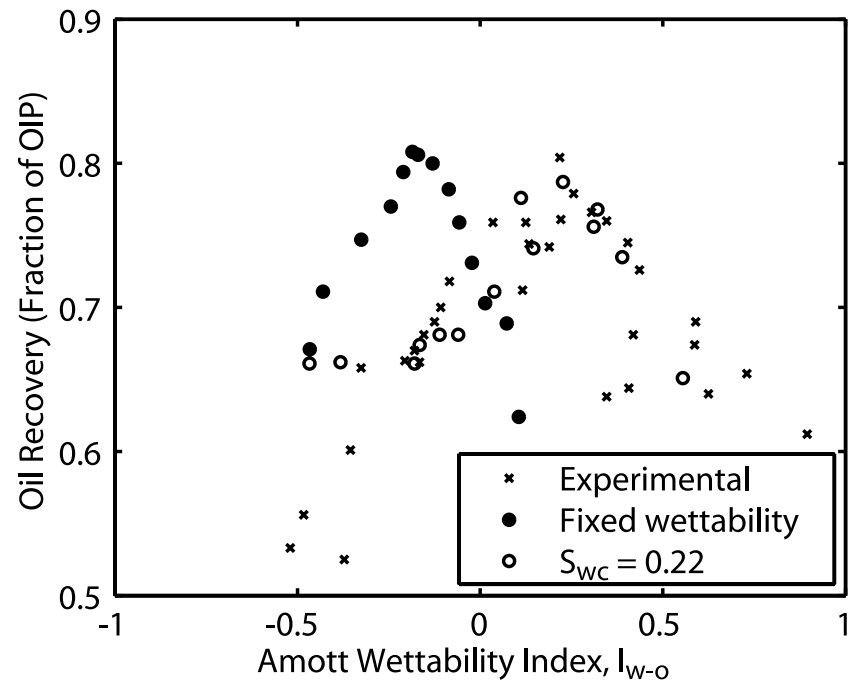

Figure 13. Recovery as a function of Amott wettability index $I_{w-o}$. Results shown are for recovery at three pore volumes injected. Predicted recoveries are shifted toward more oil wet conditions compared to experimental data.

[53] Although we are able to match the trend in recovery with $S_{w i}$ (Figure 11), the quantitative match is poor in some cases. Maximum recovery is predicted to occur at lower $S_{w i}$ and the variation in recovery with $S_{w i}$ is predicted to be less than what is observed experimentally. Recovery as a function of the Amott-Harvey water-oil index $I_{w-o}$ is shown in Figure 13. Again, the trend and maximum oil recovery are well predicted, though the predicted results are somewhat shifted toward more oil wet conditions.

[54] Our initial hypothesis that the distribution of contact angles in pores contacted by oil is independent of initial water saturation does not seem to be entirely supported when comparing predicted and experimental trends in Amott wettability indices. In Table 4 the wettability indices corresponding to Figure 11 are tabulated. It is clear that as initial water saturation increases the rock exhibits more water wet behavior than what can be attributed to just increasing $S_{w i}$. This observation is further supported by Figure 14, where more experimental data points are included. A fixed distribution of contact angles will result in a linear increase in water index with $S_{w i}$, something that is not seen experimentally. Physically this can be explained using the pore-level scenario suggested by Kovscek et al. [1993]. Initially a water film covers the rock, preventing direct contact by the oil. At a certain capillary pressure this film will rupture, allowing the rock to become more oil wet. As $S_{w i}$ increases the maximum capillary pressure during primary oil flooding is reduced, preventing some of the larger pores attaining oil wet characteristics.

[55] In the network model we assumed a constant connate water saturation of $7.9 \%$. Experimentally these low saturations could only be obtained by flooding the cores with nitrogen gas. When only flooding with brine the lowest $S_{w i}$ obtained was $22 \%$. This, combined with the fact that all the water floods were conducted on different core samples, makes it difficult to estimate accurately the true connate water saturation. What is clear is that this has significant impact on the predicted recoveries since the difference between $S_{w i}$ and $S_{w c}$ will add an additional water wet component to the system. To quantify the effect of reduced oil wetness at higher $S_{w i}$ and that $S_{w c}$ might in fact be higher than the $7.9 \%$ assumed, the wetting states of 14 experimental core samples were matched using the methodology outlined in Figure 1, keeping $S_{w c}=0.22$ for cases where $S_{w i}>0.22$, and $S_{w c}=S_{w i}$ for the rest. After primary oil flooding we assigned a target volume fraction of oil-filled pores and throats that would become oil wet. The smallest oil-filled pores were made oil wet with an intrinsic contact angle randomly distributed between $85^{\circ}$ and some upper bound. Oil-filled throats connected to oil wet pores were made oil wet with the same distribution of contact angles with a probability equal to the target oil wet fraction. Progressively larger oil-filled pores were made oil wet until the target fraction was reached. The remaining oil-filled pores and throats were assigned intrinsic contact angles uniformly between $50^{\circ}$ and $60^{\circ}$. We varied the upper bound on the oil wet contact angle distribution along with the oil wet fraction to better match the experimental Amott wetta-
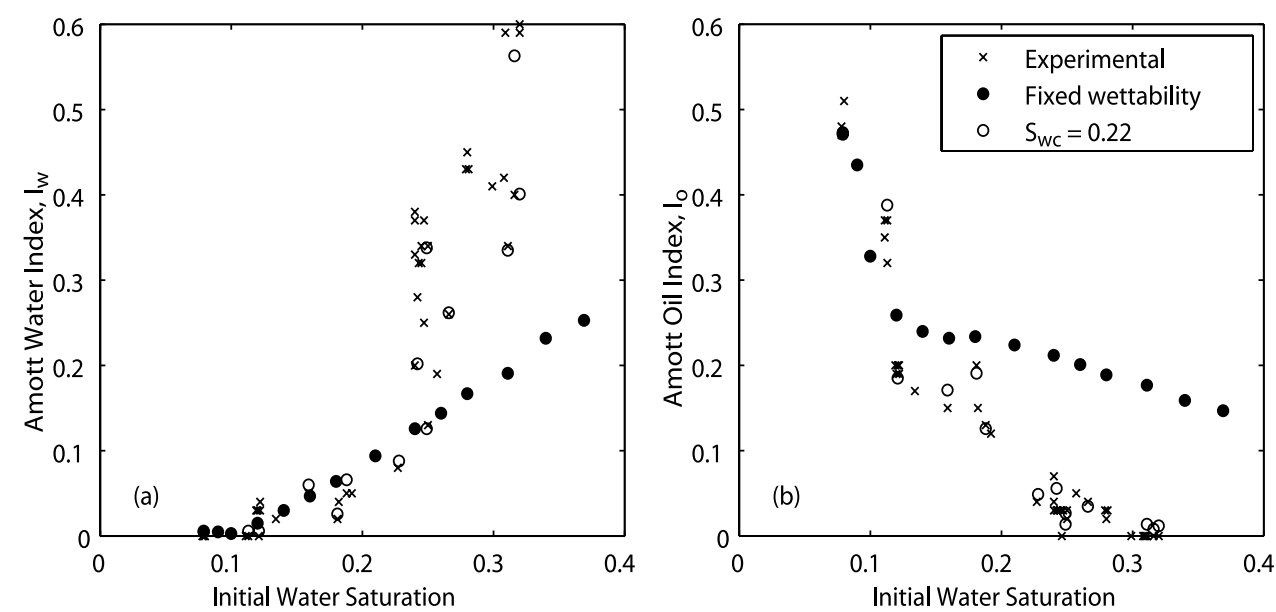

Figure 14. Influence of initial water saturation on Amott (a) water and (b) oil indices. From the experimental water indices it is clear that variation in wettability can not only be attributed to variation in $S_{w i}$. 


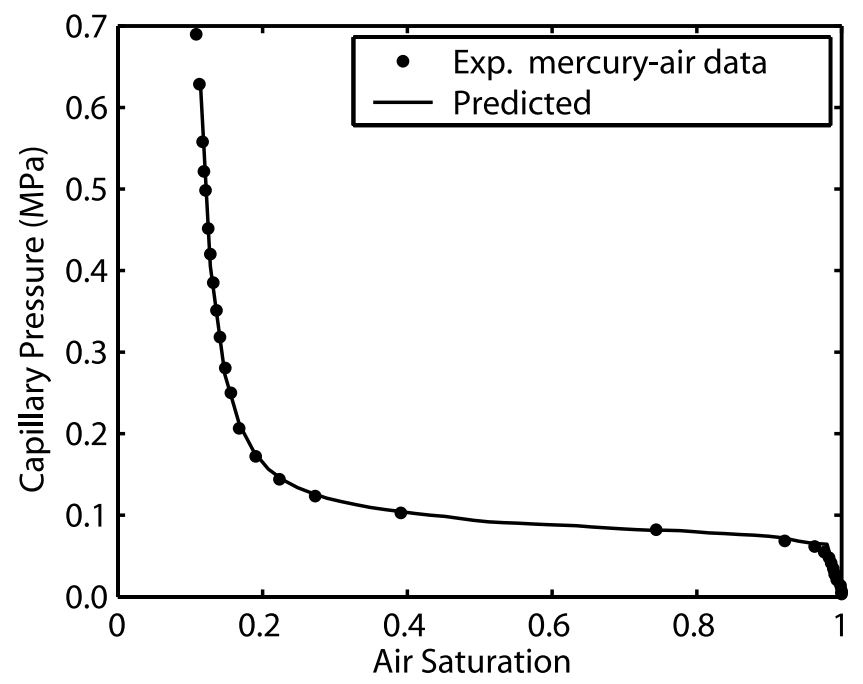

Figure 15. Comparison between experimental and matched mercury-air capillary pressure for a reservoir sandstone. Mercury will enter both microporosity and clay-bound porosity. This porosity is not explicitly included in the network model, and as a result, capillary pressures corresponding to $S_{w}<0.11$ will not be matched.

bility indices; the results are shown in Table 4 and Figure 14. The upper bound of the contact angle distribution was reduced from $120^{\circ}$ at $S_{w i}=0.08$ to $106^{\circ}$ at $S_{w i}=0.31$. The oil wet fraction was reduced from 1 to 0.76 for the same values of $S_{w i}$. Predicted recoveries are shown in Figure 13, and there now is close agreement with the experimental results.

[56] The calculated Amott water-oil index will also be significantly influenced by the choice of wettability hysteresis model (difference between advancing and receding contact angle). The Morrow [1975] model used, as illustrated in Figure 4, was developed using an ideal system. We will, however, not investigate this any further as it does not affect the overall understanding of the problem.

\subsection{Mixed Wet Reservoir Sandstone}

[57] In the petroleum industry multiphase flow experiments are routinely performed on cores from hydrocarbon bearing reservoirs. Capillary pressure data are not usually available from steady state measurements but rather found from centrifuge or mercury intrusion experiments. In both cases only pressures from the forced displacements are typically available. In this section we will use mercury-air capillary pressures to modify the network and then predict relative permeability. Though only available for the primary flooding cycle, this data has the benefit of not being influenced by wettability characterization issues as the mercury-air contact angle is assumed fixed at $120^{\circ}$ with an interfacial tension of $0.48 \mathrm{~N} / \mathrm{m}$.

[58] The capillary pressure response of the network model was matched to experimental data for a reservoir sandstone using the same procedure that was described for the sand pack, and the results are shown in Figure 15. Since no spontaneous displacement data were available, the pore body size distribution was determined by maintaining the pore body to throat radius aspect ratio from the original
Berea network. Since mercury will enter both micro and clay bound porosity, this method will not help in determining the connate water saturation. A realistic threshold radius is thus needed to determine what fraction of the pore space is accessible, effectively determining the minimum throat radius. A choice of $1 \mu \mathrm{m}$ would seem to be a realistic value in this case, resulting in $S_{w c}=0.11$. The absolute permeability was predicted to be $8.03 \times 10^{-13} \mathrm{~m}^{2}$ which compares well to the experimental value of $7.40 \times 10^{-13} \mathrm{~m}^{2}$. Mercury intrusion and steady state flow experiments are not conducted on the same cores, hence the connate water saturation might be significantly different. The nonaccessible porosity is therefore subsequently adjusted such that $S_{w c}$ in the network model is consistent with experimental data.

[59] Water flooding adds the additional complexity of wettability characterization. The Amott water index is broadly controlled by adjusting the oil wet fraction, whereas the residual oil saturation and oil index is more affected by the distribution of intrinsic contact angles in the oil wet pores. A distribution toward strongly oil wet conditions will result in low residual oil saturation as oil layers remain stable, allowing oil to escape even at low saturation. Conversely, a distribution toward more neutral conditions will result in more residual oil, as well as a lower oil index since a higher proportion of the pores will have receding contact angles less than $90^{\circ}$. The distribution of contact angles in the water wet fraction has less influence on the predicted results, though a distribution toward more strongly water wet conditions will result in poorer oil connectivity as snap-off events become more dominant.

[60] Using cryoscanning electron microscopy (cryoSEM) it is possible to visualize the distribution of oil and water at the pore scale [Fassi-Fihri et al., 1991; Durand and Rosenberg, 1998]. For carbonates there is some justification for suggesting that the pore size is important in determining what pores become oil wet. The cryo-SEM studies did, however, find that it was the larger pores that became oil wet in contrast to the previously mentioned pore-level scenario for mixed wetting [Kovscek et al., 1993] that suggested that the larger pores should remain water wet. For sandstone cores, however, it was found that the presence of clay minerals, in particular kaolinite, was far more likely to make a pore oil wet than its size. The distribution of these clay minerals might very well not be related to

Table 5. Fluid and Rock Properties Used in Predictions for a Mixed Wet Reservoir Sandstone

\begin{tabular}{ll}
\hline \multicolumn{1}{c}{ Property } & Value \\
\hline Interfacial tension $\sigma, 10^{-3} \mathrm{~N} / \mathrm{m}$ & 30.0 \\
Water viscosity $\mu_{w}, 10^{-3} \mathrm{~Pa} / \mathrm{s}$ & 1.05 \\
Oil viscosity $\mu_{o}, 10^{-3} \mathrm{~Pa} / \mathrm{s}$ & 1.39 \\
Porosity & 0.27 \\
Connate water saturation & 0.34 \\
Water wet contact angles, $\theta_{i}(\mathrm{deg})$ & $0-60$ \\
Oil wet fraction by pore volume & \\
$\quad r_{\text {min }}$ correlation & 0.53 \\
$\quad r_{\text {max }}$ correlation & 0.63 \\
spatial correlation & 0.43 \\
Oil wet contact angles $\theta_{i}$, deg & \\
$\quad r_{\text {min }}$ correlation & $70-110$ \\
$r_{\text {max }}$ correlation & $140-180$ \\
$\quad$ Spatial correlation & $100-160$ \\
\hline
\end{tabular}



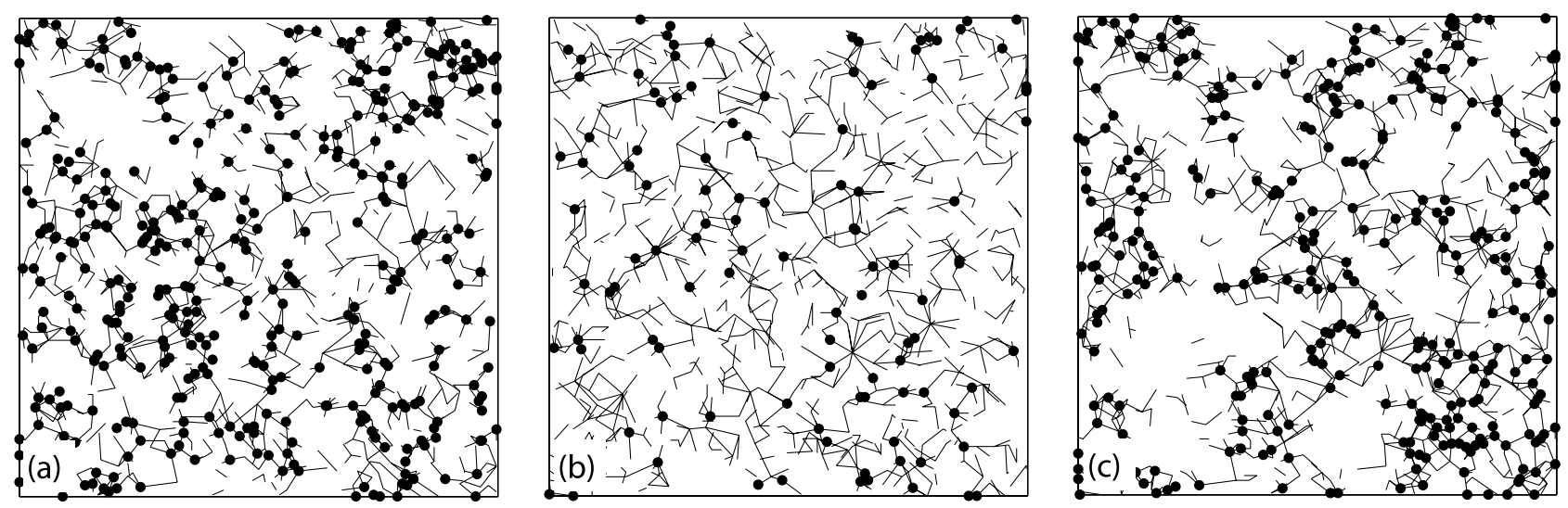

Figure 16. Distribution of water-filled elements during water flooding at $S_{w}=0.5$ with oil wet elements correlated by (a) maximum pore radius, (b) minimum pore radius, and (c) spatial correlation. The saturation shown is toward the end of spontaneous displacement. Since water wet elements are generally filled first, phase connectivity during water flooding is improved if there is spatial correlation in oil wet elements.

the pore size. In this section we will therefore test three approaches to assigning wettability. In addition to the previous approach where the smallest pores become oil wet, we will also make the largest pores oil wet. Finally we will assign oil wet elements in spatially correlated patches, as suggested by Durand and Rosenberg [1998]. This is done by randomly selecting some oil invaded pore regardless of size. All oil invaded pores within a defined correlation length are then made oil wet. Oil-filled throats connected to oil wet pores are also made oil wet. This process continues until a defined oil wet fraction is achieved. Since the oil wet elements are no deterministically defined, it becomes increasingly important to compare predictions only after multiple realizations have been simulated, minimizing the effect of nonuniqueness.

[61] Following aging the sandstone exhibited mixed wet characteristics with an Amott water index of 0.55, indicating that about half the pore space had become oil wet. No oil index was measured. Fluid and rock properties used in the predictions are given in Table 5. In Figure 16 the water distribution in a section the network model at $S_{w}=0.5$ is shown for the different wettability characterization schemes with the corresponding relative permeability curves shown in Figure 17. The saturation shown is toward the end of the spontaneous displacement and most of the remaining oilfilled elements are thus either trapped or oil wet. In both cases where oil wet elements are correlated to their inscribed radius (indicated as $r_{\min }$ and $r_{\max }$ ) there is little continuity in completely water-filled elements. As water repeatedly has to flow through layers in order to connect from inlet to outlet, the water relative permeability will stay very low until water-filled elements start to connect across the model. This behavior is very similar to that described in the previous section when $S_{w i}>S_{w c}$. In the case where the smallest pores become oil wet, the oil relative permeability is reduced very quickly as the larger pores, which are also
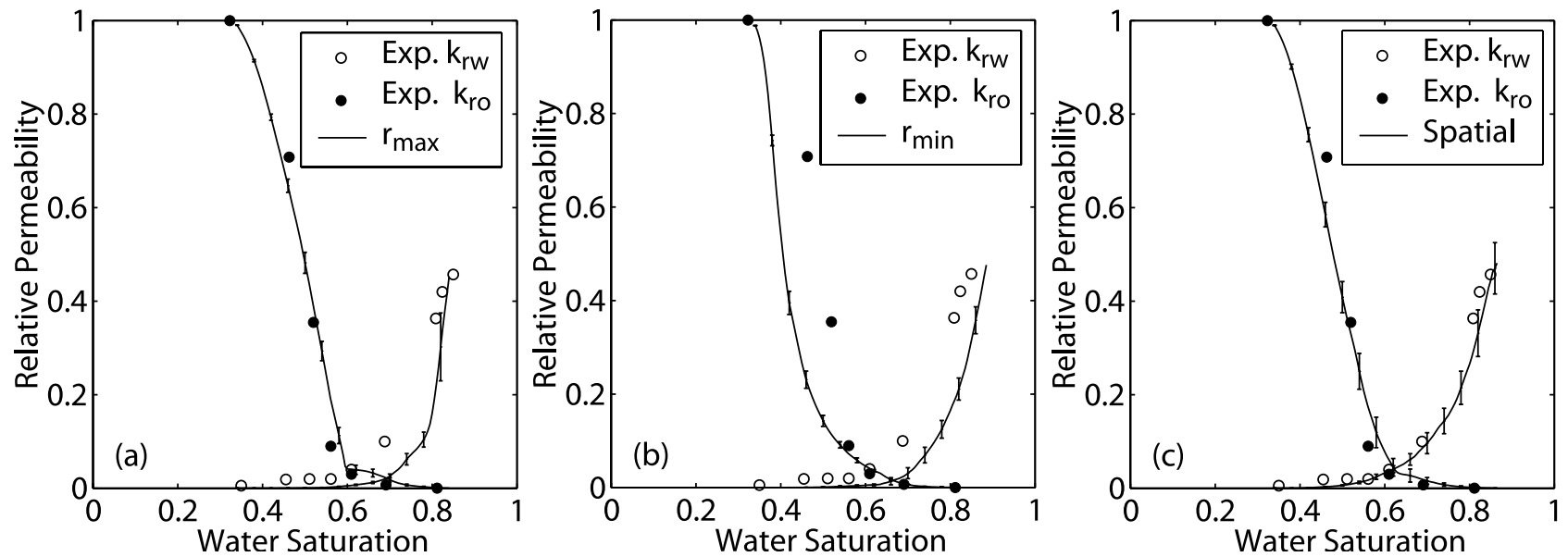

Figure 17. Comparison between experimental and predicted relative permeability for a reservoir sandstone. The experimental wetting state is fairly well predicted in all cases, and the only difference between the cases is how wettability is characterized at the pore scale. (a) The larger pores become preferentially oil wet. (b) The smaller pores become preferentially oil wet. (c) The oil wet pores are spatially correlated. 
Table 6. Predicted Amott Wettability Indices for a Mixed Wet Reservoir Sandstone

\begin{tabular}{lcclll}
\hline & \multicolumn{2}{c}{ Water } & & \multicolumn{2}{c}{ Oil } \\
\cline { 2 - 3 } \cline { 5 - 6 } & $\begin{array}{c}\text { Amott } \\
\text { Index }\end{array}$ & $\begin{array}{c}\text { Standard } \\
\text { Deviation }\end{array}$ & & $\begin{array}{c}\text { Amott } \\
\text { Index }\end{array}$ & $\begin{array}{c}\text { Standard } \\
\text { Deviation }\end{array}$ \\
\hline Experimental & 0.55 & - & & - & - \\
$r_{\text {max }}$ correlation & 0.51 & 0.01 & & 0.46 & 0.01 \\
$r_{\text {min }}$ correlation & 0.54 & 0.01 & & 0.00 & 0.00 \\
Spatial correlation & 0.56 & 0.08 & & 0.32 & 0.08 \\
\hline
\end{tabular}

the most conductive, are filled by water early in the displacement. Spatially correlating the oil wet elements, with a correlation length of about 7 pores, creates more continuity through the oil wet pores, making it more likely for water to be able to flow from inlet to outlet in completely water-filled elements. The result is a more gradual increase in water relative permeability, consistent with the experimental data. There is necessarily going to be more statistical variation between the different realizations when using a spatial correlation approach, evident from the larger error bars in Figure 17. All the predicted Amott water indices are consistent with the experimental values (Table 6). There is more variation in the predicted oil indices as there were no experimental data to compare against.

\subsection{Oil Wet Reservoir Sandstone}

[62] The final sandstone data set had steady state relative permeabilities measured on 2 core samples with experimental permeabilities of 248 and $328 \mathrm{mD}$. Since no mercury injection data were available, primary oil flooding capillary pressure had to be used for the network modification process (Figure 18). This was a centrifuge experiment performed on a different core sample from those used for relative permeability measurements, hence the difference in connate water saturation. Uncertainties in interfacial tension and contact angles meant that no reliable estimate of absolute permeability could be made. Rather than being strongly water wet during primary oil flooding, the core exhibited mixed wet characteristics with Amott water and oil indices of 0.33 and 0 . Though this is consistent with a uniform distribution of receding contact angles between 10 and $40^{\circ}$, there still is quite a large degree of uncertainty in the exact wetting state.

[63] Following wettability alteration the core exhibited mainly oil wet characteristics with Amott water and oil indices of 0.00 and 0.14 . The low residual oil saturation is also an indication of an oil wet system, a result of oil being able to escape through layers. The comparison between experimental and predicted relative permeability is good (Figure 18). The predictions were made with $S_{w i}=0.03$ and intrinsic contact angles distributed at random uniformly between $70^{\circ}$ and $122^{\circ}$. The statistical fluctuations between realizations are less than for mixed wet samples as all pores contacted by oil are assumed to be oil wet. The predicted Amott indices of 0.00 and 0.15 , with standard deviations of 0 and 0.01 , for water and oil respectively are also in good agreement with experimental values. Fluid and rock properties are given in Table 7.

\section{Conclusions}

[64] Using a geological realistic network initially developed to represent Berea sandstone we successfully predicted flow properties for several data sets including a sand pack and two reservoir sandstones. This suggests that a realistic network topology combined with network properties tuned to experimental data such as mercury injection capillary pressure is sufficient to predict single and multiphase properties for a wide range of porous media. For this work we have only used a single network constructed from Berea sandstone. To improve confidence in predictions from a wider range of rocks it would be preferable to have a library of networks representing different rock types. The network that most closely matches the geological structure of the sample of interest would then be chosen for modeling studies.

[65] During water flooding relative permeability is strongly affected by wettability. The trend in oil recovery from a series of mixed wet Berea sandstone cores [Jadhunandan and Morrow, 1995] was well predicted by closely reproducing the experimental conditions such as $S_{w i}$ and saturation history. The focus of this work is not
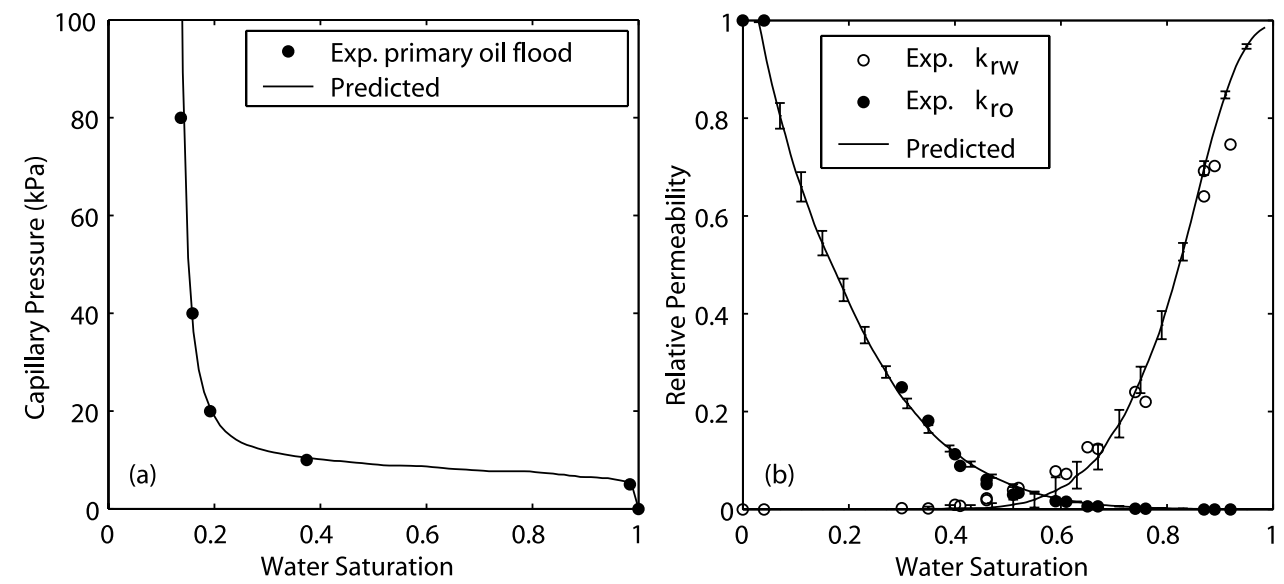

Figure 18. Comparison between predicted and experimental properties for an oil wet reservoir sandstone. (a) Centrifuge primary oil flooding capillary pressures were used to modify the network. (b) Predicted water flooding relative permeability is compared to experimental steady state data. Low residual oil saturation is a good indication oil wet characteristics. 
Table 7. Fluid and Rock Properties Used in Predictions for an Oil Wet Reservoir Sandstone

\begin{tabular}{ll}
\hline \multicolumn{1}{c}{ Property } & \multicolumn{1}{c}{ Value } \\
\hline Interfacial tension $\sigma, 10^{-3} \mathrm{~N} / \mathrm{m}$ & 51.8 \\
Water viscosity $\mu_{w}, 10^{-3} \mathrm{~Pa} / \mathrm{s}$ & 1.00 \\
Oil viscosity, $\mu_{o}\left(10^{-3} \mathrm{~Pa} / \mathrm{s}\right)$ & 0.29 \\
Porosity & 0.15 \\
Connate water saturation & 0.00 \\
Initial water saturation & 0.03 \\
Contact angles during primary oil flooding $\theta_{r}, \mathrm{deg}$ & $10-40$ \\
Contact angles during water flooding, $\theta_{i}, \mathrm{deg}$ & $70-122$
\end{tabular}

so much on obtaining a quantitative match with the experiments, but rather on understanding the pore-scale processes that produce these surprising experimental recovery trends. However, by modifying our assumptions about the experimental conditions, a quantitative match was obtained.

[66] Wettability characterization for mixed wet systems is perhaps the most uncertain part of what has been investigated in this work. Though theoretical models have been suggested in the literature [Kovscek et al., 1993], these do not always satisfactorily describe what is observed experimentally, both in terms of observed transport properties and from pore-scale imaging [Fassi-Fihri et al., 1991; Durand and Rosenberg, 1998]. In the case of the mixed wet sandstone samples we suggest that good predictions require the oil wet pores to be spatially correlated. This ensured that the predicted relative permeability was in better agreement with experimental data than when correlating the oil wet pores based on size only.

\section{Appendix A}

\section{A1. Calculation of Corner Half Angles}

[67] For a triangle with a given shape factor the corner half angles $\beta$ can take on a range of values where $\beta_{1} \leq \beta_{2} \leq$ $\beta_{3}$. First $\beta_{2}$ is chosen randomly within the allowed bound

$$
\beta_{2, \min }=\operatorname{atan}\left\{\frac{2}{\sqrt{3}} \cos \left[\frac{\operatorname{acos}(-12 \sqrt{3} G)}{3}+\frac{4 \pi}{3}\right]\right\}
$$

and

$$
\beta_{2, \max }=\operatorname{atan}\left\{\frac{2}{\sqrt{3}} \cos \left[\frac{\operatorname{acos}(-12 \sqrt{3} G)}{3}\right]\right\},
$$

with $\beta_{1}$ subsequently given by

$$
\beta_{1}=-\frac{1}{2} \beta_{2}+\frac{1}{2} \operatorname{asin}\left(\frac{\tan \beta_{2}+4 G}{\tan \beta_{2}-4 G}\right),
$$

and finally $\beta_{3}=\pi / 2-\beta_{1}-\beta_{2}$ [Patzek, 2001].

\section{A2. Calculation of Capillary Entry Pressure During Forced Oil Flooding Using the MS-P Method}

[68] Consider the cross section of a polygonal-shaped element, of which one corner is shown in Figure A1. A small displacement $\mathrm{d} x$ of the AMs must be balanced by the change in surface free energy,

$$
P_{c} A_{e f f}=\left(L_{o w} \sigma_{o w}+L_{o s} \sigma_{o s}+L_{o s} \sigma_{w s}\right) \mathrm{d} x
$$

where $A_{\text {eff }}$ is effective area occupied by oil, $L_{o s}$ is the length of the oil-surface interface and $L_{o w}$ is the length of the oilwater interface. A horizontal force balance gives the Young equation,

$$
\sigma_{o s}=\sigma_{w s}+\sigma_{o w} \cos \theta_{o w r}
$$

that allows us to simplify equation (A4),

$$
\frac{P_{c}}{\sigma_{o w}}=\frac{1}{R}=\frac{L_{o w}+L_{o s} \cos \theta_{o w r}}{A_{\text {eff }}},
$$

where $R$ is the radius of curvature. The interface lengths can be determined from elementary geometry,

$$
\begin{gathered}
A_{\text {eff }}=A-R^{2} \sum_{i=1}^{n}\left[\frac{\cos \theta_{\mathrm{r}} \cos \left(\theta_{r}+\beta_{i}\right)}{\sin \beta_{i}}+\theta_{r}+\beta_{i}-\frac{\pi}{2}\right]=\frac{r^{2}}{4 G}-R^{2} S_{1}, \\
L_{o s}=\frac{r}{2 G}-2 R \sum_{i=1}^{n} \frac{\cos \left(\theta_{r}+\beta_{i}\right)}{\sin \beta_{i}}=\frac{r}{2 G}-2 R S_{2}, \\
L_{o w}=2 R \sum_{i=1}^{n}\left(\frac{\pi}{2}-\theta_{r}-\beta_{i}\right)=R S_{3},
\end{gathered}
$$

where $n$ is the total number of corners containing AMs $(\beta<$ $\left.\pi / 2-\theta_{r}\right)$ and $A=r^{2} /(4 G)$ is the total polygon area. A quadratic expression for the curvature radius is then given by,

$$
R=\frac{r \cos \theta_{r}\left(-1 \pm \sqrt{1+\frac{4 G D}{\cos ^{2} \theta_{r}}}\right)}{4 G D},
$$

$$
D=S_{1}-2 S_{2} \cos \theta_{r}+S_{3} .
$$

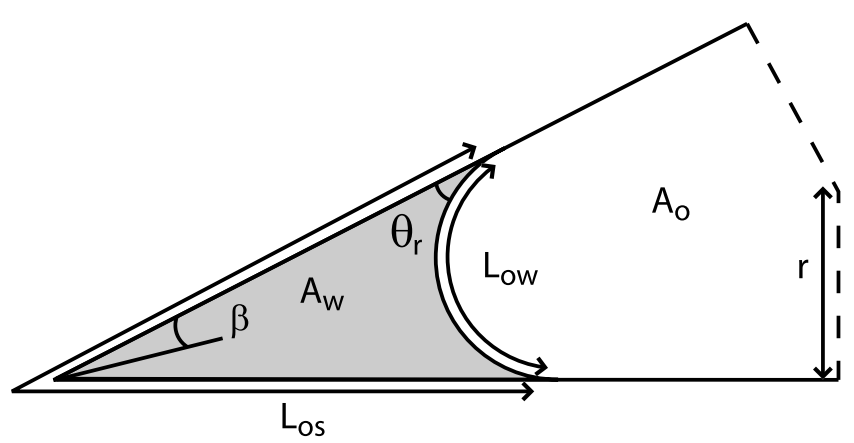

Figure A1. Cross-sectional view of a single corner in a polygonal-shaped element. 

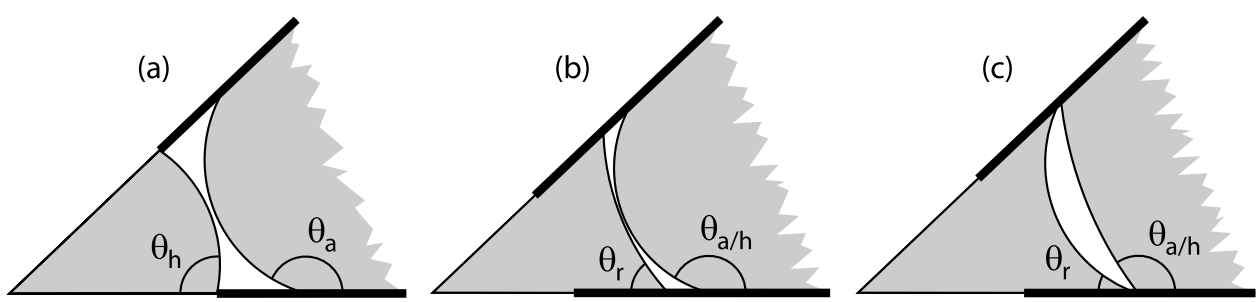

Figure A2. Possible layer configurations that might occur in network elements. (a) During secondary water flooding, the layer will collapse once the two interfaces meet at the crest of the interface. (b) During higher-order flooding cycles, several more layer configurations are available. If water either in the center or in the layers is trapped, the capillary pressure across the interfaces might not be equal. (c) Depending on the capillary pressures the layer might collapse as the interfaces meet at the base rather than at the crest.

The solution for curvature radius that is less than the inscribed radius is valid. The capillary entry pressure thus given by [Øren et al., 1998],

$$
P_{c}=\frac{\sigma \cos \theta_{r}(1+2 \sqrt{\pi G})}{r} F_{d}\left(\theta_{r}, G, \beta\right)
$$

where $F_{d}$ is a dimensionless correction factor for wetting fluid that might be retained in the corners,

$$
F_{d}\left(\theta_{r}, G, \beta\right)=\frac{1+\sqrt{1+\frac{4 G D}{\cos ^{2} \theta_{r}}}}{(1+2 \sqrt{\pi G})} .
$$

In the case of a circular tube where there are no corners, $F_{d}$ will be 1 and equation (A13) will simplify to equation (3).

\section{A3. Calculation of Capillary Entry Pressure During Water Flooding Using MS-P Method}

[69] During spontaneous (positive capillary pressure) piston-like displacement the capillary entry pressure is found by calculating the force balance acting on the interface. Since the hinging contact angle is a function of capillary pressure, the following equations are solved iteratively to obtain the solution for the curvature radius corresponding to the capillary entry pressure [Oren et al., 1998],

$$
\begin{gathered}
R=\frac{A_{\text {eff }}}{L_{o w}+L_{o s} \cos \theta_{a}}, \\
A_{\text {eff }}=A-R^{2} \sum_{i=1}^{n}\left[\frac{\cos \theta_{h, i} \cos \left(\theta_{h, i}+\beta_{i}\right)}{\sin \beta_{i}}+\theta_{h, i}+\beta_{i}-\frac{\pi}{2}\right], \\
L_{o s}=\frac{r}{2 G}-2 \sum_{i=1}^{n} b_{i}, \\
L_{o w}=2 R \sum_{i=1}^{n} \operatorname{asin}\left(\frac{b_{i} \sin \beta_{i}}{R}\right) .
\end{gathered}
$$

If the hinging contact angles have all reached the limiting advancing value the expression for the capillary entry pressure will be the same as that during primary oil flooding with the receding contact angle replaced by the advancing one. In the case where one or more of the interfaces have reached the advancing contact angle the expression for $b_{i}$ equation (4) is simply replaced by

$$
b_{i}=R \frac{\cos \left(\theta_{a}+\beta_{i}\right)}{\sin \beta_{i}} .
$$

[70] From these expressions it is clear that spontaneous displacement might occur for advancing contact angles greater than $90^{\circ}$, with the maximum angle given by,

$$
\cos \theta_{a, \max } \approx \frac{-4 G \sum_{i=1}^{n} \cos \left(\theta_{r}+\beta_{i}\right)}{\frac{r}{R_{\min }}-\cos \theta_{r}+12 G \sin \theta_{r}} .
$$

During forced water invasion (negative capillary pressure) the absolute entry pressure is simply given by equation (A12) with $\theta_{r}$ replaced by $\pi-\theta_{a}$.

\section{A4. Calculation of Capillary Pressure for the Collapse and Reformation of Oil Layers}

[71] If none of the water is trapped, but both the inner $\theta_{i h, j}$ and outer $\theta_{o h, j}$ contact angles for a given corner $j$ are hinging, as depicted in Figure A2. The collapsing capillary pressure can be found by iteratively solving,

$$
\begin{gathered}
\frac{\cos \theta_{i h, j}-\cos \theta_{o h, j}}{\sin \beta}=2, \\
\cos \left(\cos \theta_{i h, j}+\beta_{j}\right)=\frac{b_{i, j} \sin \beta_{j}}{R}, \\
\cos \left(\cos \theta_{o h, j}-\beta_{j}\right)=\frac{b_{o, j} \sin \beta_{j}}{R},
\end{gathered}
$$

where $b_{i, j}$ and $b_{o, j}$ are the pinned apex distances for the inner and outer interfaces.

[72] If water in the corner is trapped, the inner interface has a constant radius of curvature $R_{i}$ and inner hinging contact angle $\theta_{i h, j}$, fixed in position the moment water got 
trapped. If the outer interface is not hinging, the oil layer will collapse at a radius of curvature,

$$
R=\frac{R_{i}\left(\cos \theta_{i h_{j}}-\sin \beta_{j}\right)}{\cos \theta_{a}+\sin \beta_{j}},
$$

whereas if the outer interface is hinging, the radius of curvature can be found by iteratively solving equation (A22) in combination with equation (A23), replacing $\theta_{a}$ with $\theta_{o h, j}$.

[73] Similarly, if water in the center is trapped, the outer interface has a constant radius of curvature $R_{o}$. The collapsing radius of curvature is found iteratively by solving equation (A21) in combination with,

$$
R=\frac{R_{o}\left(\cos \theta_{a}+\sin \beta_{j}\right)}{\cos \theta_{i h, j}-\sin \beta_{j}} .
$$

If outer interface in hinging, replace $\theta_{a}$ with $\theta_{o h, j}$.

[74] In some cases the layer might collapse once the interfaces touch at the base rather than the crest of the interface (Figure A2c). This is possible if water in the corner is trapped with $\theta_{i h, j}<\pi-\theta_{a}$. The radius of curvature is then given by,

$$
R=\frac{b_{i, j} \sin \beta_{j}}{\cos \left(\theta_{a}-\beta_{j}\right)} .
$$

\section{A5. Empirical Expressions for Flow in Corners and Layers}

[75] There are a number of variables affecting the shape of the corners and layers (Figure A3): half angle $\beta$, contact angles $\theta$ and meniscus apex distances $b$. The boundary condition for the fluid interface is clearly very important. Here we assume infinite surface shear viscosity (no-slip boundary condition) which should be valid for a surfactantladen water-oil interface.

[76] For flow in corners we use the correlation proposed by Øren et al. [1998], given by

$$
\begin{gathered}
A_{c}=\left(\frac{b_{i} \sin \beta}{\cos \left(\theta_{i}+\beta\right)}\right)^{2}\left(\frac{\cos \theta_{i} \cos \left(\theta_{i}+\beta\right)}{\sin \beta}+\theta_{i}+\beta-\frac{\pi}{2}\right), \\
G_{c}=\frac{A_{c}}{4 b_{i}^{2}\left(1-\frac{\sin \beta}{\cos \left(\theta_{i}+\beta\right)}\left(\theta_{i}+\beta-\frac{\pi}{2}\right)\right)^{2}}, \\
G^{*}=\frac{\sin \beta \cos \beta}{4(1+\sin \beta)^{2}} \\
C=0.364+0.28 \frac{G^{*}}{G_{c}} \\
g_{p c}=C \frac{A_{c}^{2} G_{c}}{\mu_{p}}
\end{gathered}
$$

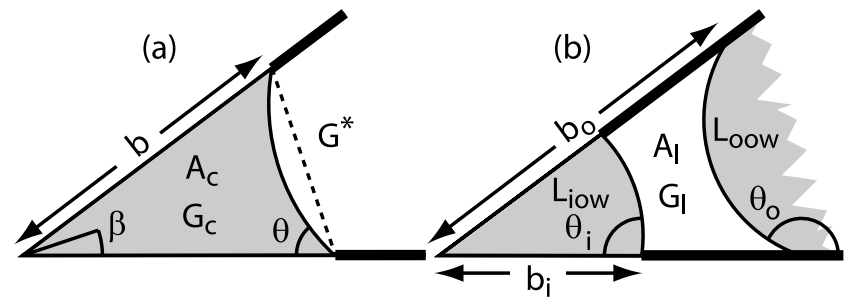

Figure A3. Corner configurations along with parameters affecting the shape. (a) Flow in corner. (b) Flow in layer.

where $G_{c}$ is the shape factor for the corner section containing the fluid, $G^{*}$ is the shape factor without any curvature on the fluid interface and $A_{c}$ is the corner area. The performance of the correlation was tested using a 2-D finite element code for incompressible laminar flow. For a variety of corner configurations it was found to predict the conductance well within a ten \% error margin.

[77] Predicting the conductance of oil layers is more difficult since there are two interfaces to include (Figure A3b). Numerical simulations were once again conducted, with 1035 different configurations investigated. The outer contact angle $\theta_{o}$ was varied between $120^{\circ}$ and $180^{\circ}$, the meniscus apex distance ratio between 0.01 and 0.6 and layer shape factor between 0.002 and 0.048 , resulting in a variation of corner half angles from $3^{\circ}$ to $62^{\circ}$. No correlation in the literature was found to predict the hydraulic conductance adequately. On the basis of these simulations we therefore suggest the following correlation:

$$
\tilde{b}_{i}=\frac{b_{i}}{b_{o}}
$$

$$
\begin{gathered}
\tilde{A}_{o}=\left(\frac{\sin \beta}{\cos \left(\theta_{o}-\beta\right)}\right)^{2}\left(\frac{\cos \theta_{o} \cos \left(\theta_{o}-\beta\right)}{\sin \beta}-\theta_{o}+\beta+\frac{\pi}{2}\right), \\
\tilde{A}_{i}=\left(\frac{\tilde{b}_{i} \sin \beta}{\cos \left(\theta_{i}+\beta\right)}\right)^{2}\left(\frac{\cos \theta_{i} \cos \left(\theta_{i}+\beta\right)}{\sin \beta}+\theta_{i}+\beta-\frac{\pi}{2}\right),
\end{gathered}
$$

$$
\tilde{A}_{l}=\tilde{A}_{o}-\tilde{A}_{i},
$$

$$
\tilde{L}_{\text {oow }}=\frac{2 \sin \beta}{\cos \left(\theta_{o}-\beta\right)}\left(\frac{\pi}{2}-\theta_{o}+\beta\right),
$$

$$
\tilde{L}_{\text {iow }}=\frac{2 \tilde{b}_{i} \sin \beta}{\cos \left(\theta_{i}+\beta\right)}\left(\frac{\pi}{2}-\theta_{i}-\beta\right),
$$

$$
G_{l}=\frac{\tilde{A}_{l}}{\left(\tilde{L}_{\text {oow }}+\tilde{L}_{i o w}+2\left(1-\tilde{b}_{i}\right)\right)^{2}},
$$

$$
\ln \tilde{g}_{l}=a_{1} \ln ^{2}\left(\tilde{A}_{l}^{3} G_{l}\right)+a_{2} \ln \left(\tilde{A}_{l}^{3} G_{l}\right)+a_{3},
$$

$$
g_{p l}=\frac{b_{o}^{4} \tilde{g}_{l}}{\mu_{p}},
$$




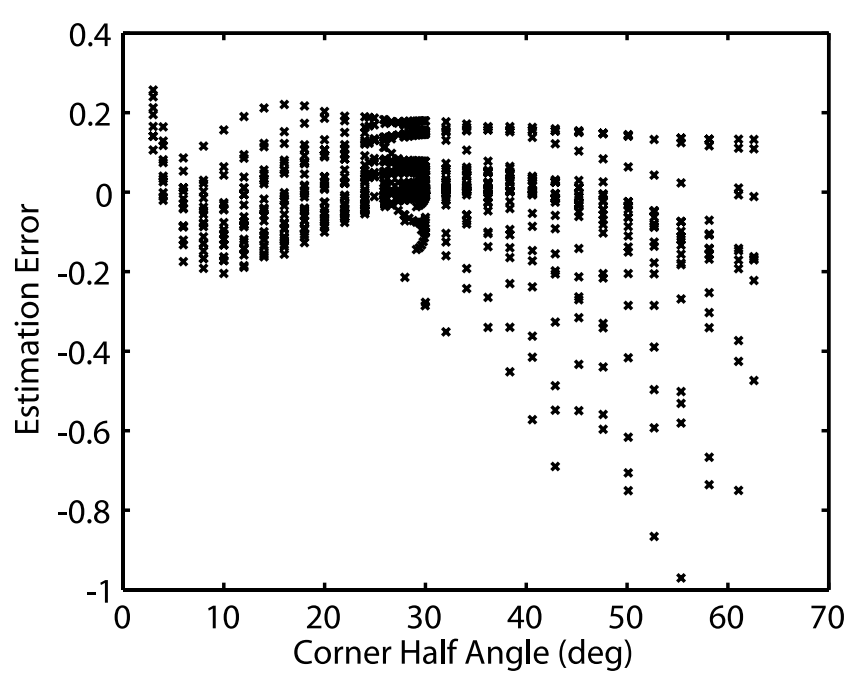

Figure A4. Performances of proposed layer conductance correlations when compared to numerical finite element simulations.

where $\tilde{A}_{l}$ is the dimensionless area of the layer, $\tilde{A}_{o}$ and $\tilde{A}_{i}$ are the outer and inner dimensionless corner areas, $\tilde{L}_{\text {oow }}$ and $\tilde{L}_{\text {iow }}$ are the dimensionless oil-water interface lengths, $G_{l}$ is the layer shape factor and $\tilde{g}_{l}$ is the dimensionless layer conductance. The constants $a_{1}$ to $a_{3}$ are found from a polynomial fit to the numerical results, with $a_{1}=-0.02401$, $a_{2}=0.2840$ and $a_{3}=-2.953$. The performance of the correlation, when compared to numerical simulation results, is shown in Figure A4. The mean absolute error is $8.7 \%$ with the largest errors occurring in the wider corners. This is not such a problem as the combined layer conductance for a network element will typically be dominated by the sharpest corner.

[78] Acknowledgments. The members of the Imperial College Consortium on Pore-Scale Modeling (BHP, Gaz de France, JNOC, PDVSAIntevep, Schlumberger, Shell, Statoil, the UK Department of Trade and Industry and the EPSRC) are thanked for their financial support. We also thank Pål-Eric Øren (Statoil) for sharing his Berea network data with us. Shell and Statoil are also thanked for providing us with experimental data. The code used to generate the results in this paper together with example input files can be downloaded at http://www.ese.ic.ac.uk/general.php? $\mathrm{GenID}=407$.

\section{References}

Adler, P. M., and J. F. Thovert (1998), Real porous media: Local geometry and macroscopic properties, Appl. Mech. Rev., 51, 537-585.

Al-Futaisi, A., and T. W. Patzek (2003), Impact of wettability alteration on two-phase flow characteristics of sandstones: A quasi-static description, Water Resour. Res., 39(2), 1042, doi:10.1029/2002WR001366.

Anderson, W. G. (1986a), Wettability literature survey-part 1: Rock-oilbrine interactions and the effects of core handling on wettability, J. Pet. Technol., 38, 1125-1144.

Anderson, W. G. (1986b), Wettability literature survey_part 2: Wettability measurement, J. Pet. Technol., 38, 1246-1262.

Bakke, S., and P. E. Øren (1997), 3-D pore-scale modelling of sandstones and flow simulations in the pore networks, SPE J., 2, 136-149.

Blunt, M. J. (1998), Physically-based network modeling of multiphase flow in intermediate-wet porous media, J. Pet. Sci. Eng., 20, 117-125.

Blunt, M. J., and H. Scher (1995), Pore-level modeling of wetting, Phys. Rev. E, 52, 6387-6403.

Blunt, M. J., M. D. Jackson, M. Piri, and P. H. Valvatne (2002), Detailed physics, predictive capabilities and macroscopic consequences for pore-network models of multiphase flow, Adv. Water Resour., 25, $1069-1089$
Bryant, S., and M. Blunt (1992), Prediction of relative permeability in simple porous-media, Phys. Rev. A, 46, 2004-2011.

Bryant, S. L., P. R. King, and D. W. Mellor (1993a), Network model evaluation of permeability and spatial correlation in a real random sphere packing, Transp. Porous Media, 11, 53-70.

Bryant, S. L., D. W. Mellor, and C. A. Cade (1993b), Physically representative network models of transport in porous-media, AIChE J., 39, 387396.

Chatzis, I., and F. A. L. Dullien (1977), Modelling pore structures by 2-D and 3-D networks with application to sandstones, J. Can. Pet. Technol., $16,97-108$.

Combes, R., M. Robin, G. Blavier, M. Aidan, and F. Degreve (1998), Visualization of imbibition in porous media by environmental scanning electron microscopy: Application to reservoir rocks, J. Pet. Sci. Eng., 20, $133-139$.

Delerue, J. F., and E. Perrier (2002), DXSoil, a library for 3D image analysis in soil science, Comput. Geosci., 28, 1041-1050.

Dixit, A. B., S. R. McDougall, and K. S. Sorbie (1997), Pore-level investigation of relative permeability hysteresis in water-wet systems, Pap. SPE 37233, Soc. of Pet. Eng., Richardson, Tex.

Dixit, A. B., S. R. McDougall, K. S. Sorbie, and J. S. Buckley (1999), Porescale modeling of wettability effects and their influence on oil recovery, SPE Reservoir Eng. Eval. Eng., 2, 25-36.

Dullien, F. A. L. (1992), Porous Media: Fluid Transport and Pore Structure, 2nd ed., Academic, San Diego, Calif.

Dunsmuir, J. H., S. R. Ferguson, K. L. D’Amico, and J. P. Stokes (1991), $\mathrm{X}$-ray microtomography. A new tool for the characterization of porous media, Pap. SPE 22860, Soc. of Pet. Eng., Richardson, Tex.

Durand, C., and E. Rosenberg (1998), Fluid distribution in kaolinite- or illite-bearing cores: Cryo-SEM observations versus bulk measurements, J. Pet. Sci. Eng., 19, 65-72.

Dury, O. (1997), Organic pollutants in unsaturated soils: Effect of butanol as model contaminant on phase saturation and flow characteristics of a quartz sand packing, Ph.D. thesis, Swiss Fed. Inst. of Technol., Zürich.

Dury, O., U. Fischer, and R. Schulin (1998), Dependence of hydraulic and pneumatic characteristics of soils on a dissolved organic compound, J. Contam. Hydrol., 33, 39-57.

Fassi-Fihri, O., M. Robin, and E. Rosenberg (1991), Wettability studies at the pore level. A new approach by the use of Cryo-Scanning Electron Microscopy, Pap. SPE 22596, Soc. of Pet. Eng., Richardson, Tex.

Fischer, U., and M. A. Celia (1999), Prediction of relative and absolute permeabilities for gas and water from soil water retention curves using a pore-scale network model, Water Resour. Res., 35, 1089-1100.

Jackson, M. D., P. H. Valvatne, and M. J. Blunt (2003), Prediction of wettability variation and its impact on flow using pore- to reservoir-scale simulations, J. Pet. Sci. Eng., 39, 231-246.

Jadhunandan, P. P., and N. R. Morrow (1995), Effect of wettability on waterflood recovery for crude-oil brine rock systems, SPE Reservoir Eng., 10, 40-46.

Jerauld, G. R., and S. J. Salter (1990), Effect of pore-structure on hysteresis in relative permeability and capillary pressure: Pore-level modeling, Transp. Porous Media, 5, 103-151.

Kaminsky, R., and C. J. Radke (1997), Asphaltenes, water films, and wettability reversal, $S P E$ J., 2, 485-493.

Kovscek, A. R., H. Wong, and C. J. Radke (1993), A pore-level scenario for the development of mixed wettability in oil-reservoirs, AIChE J., 39, $1072-1085$.

Lenormand, R., C. Zarcone, and A. Sarr (1983), Mechanisms of the displacement of one fluid by another in a network of capillary ducts, J. Fluid Mech., 135, 337-353.

Lerdahl, T. R., P. E. Øren, and S. Bakke (2000), A predictive network model for three-phase flow in porous media, Pap. SPE 59311, Soc. of Pet. Eng., Richardson, Tex.

Lindquist, W. B., S. M. Lee, D. A. Coker, K. W. Jones, and P. Spanne (1996), Medial axis analysis of void structure in three-dimensional tomographic images of porous media, J. Geophys. Res., 101, 8297-8310.

Masalmeh, S. K. (2003), The effect of wettability heterogeneity on capillary pressure and relative permeability, J. Pet. Sci. Eng., 39, 299-408.

Mason, G., and N. R. Morrow (1991), Capillary behavior of a perfectly wetting liquid in irregular triangular tubes, J. Colloid Interface Sci., 141, 262-274.

Morrow, N. R. (1975), Effects of surface roughness on contact angle with special reference to petroleum recovery, J. Can. Pet. Technol., 14, 42-53.

Oak, M. J. (1990), Three-phase relative permeability of water-wet Berea, Pap. SPE 20183, Soc. of Pet. Eng., Richardson, Texas.

Øren, P. E., and S. Bakke (2003), Reconstruction of Berea sandstone and pore-scale modelling of wettability effects, J. Pet. Sci. Eng., 39, 177-199. 
Øren, P. E., S. Bakke, and O. J. Arntzen (1998), Extending predictive capabilities to network models, SPE J., 3, 324-336.

Patzek, T. W. (2001), Verification of a complete pore network simulator of drainage and imbibition, SPE J., 6, 144-156.

Patzek, T. W., and D. B. Silin (2001), Shape factor and hydraulic conductance in noncircular capillaries I. One-phase creeping flow, J. Colloid Interface Sci., 236, 295-304.

Rajaram, H., L. A. Ferrand, and M. A. Celia (1997), Prediction of relative permeabilities for unconsolidated soils using pore-scale network models, Water Resour. Res., 33, 43-52.

Sok, R. M., M. A. Knackstedt, A. P. Sheppard, W. V. Pinczewski, W. B. Lindquist, A. Venkatarangan, and L. Paterson (2002), Direct and stochastic generation of network models from tomographic images: Effect of topology on residual saturations, Transp. Porous Media, 46, 345-372.

Spanne, P., J. F. Thovert, C. J. Jacquin, W. B. Lindquist, K. W. Jones, and P. M. Adler (1994), Synchrotron computed microtomography of
porous-media-Topology and transports, Phys. Rev. Lett., 73, 2001 2004.

Vogel, H. J. (2000), A numerical experiment on pore size, pore connectivity, water retention, permeability, and solute transport using network models, Eur. J. Soil Sci., 51, 99-105.

Wilkinson, D., and J. F. Willemsen (1983), Invasion percolation-A new form of percolation theory, J. Phys. A Math. Gen., 16, 3365-3376.

Wise, W. R. (1992), A new insight on pore structure and permeability, Water Resour. Res., 28, 189-198.

M. J. Blunt, Department of Earth Science and Engineering, Imperial College London, London SW7 2AZ, UK. (m.blunt@imperial.ac.uk)

P. H. Valvatne, Shell International Exploration and Production B.V., Kesslerpark 1, 2288 GS Rijswijk, Netherlands. (per.valvatne@shell.com) 